San Jose State University

SJSU ScholarWorks

Master's Theses

Master's Theses and Graduate Research

Fall 2015

\title{
Formation of Organic Films on Sulfuric Acid Solutions at Upper Troposphere and Lower Stratosphere Aerosol Acidities
}

Holger Saul Perez

San Jose State University

Follow this and additional works at: https://scholarworks.sjsu.edu/etd_theses

\section{Recommended Citation}

Perez, Holger Saul, "Formation of Organic Films on Sulfuric Acid Solutions at Upper Troposphere and Lower Stratosphere Aerosol Acidities" (2015). Master's Theses. 4662.

DOI: https://doi.org/10.31979/etd.cdfj-6k26

https://scholarworks.sjsu.edu/etd_theses/4662

This Thesis is brought to you for free and open access by the Master's Theses and Graduate Research at SJSU ScholarWorks. It has been accepted for inclusion in Master's Theses by an authorized administrator of SJSU ScholarWorks. For more information, please contact scholarworks@sjsu.edu. 


\title{
FORMATION OF ORGANIC FILMS ON SULFURIC ACID SOLUTIONS AT UPPER
} TROPOSPHERE AND LOWER STRATOSPHERE AEROSOL ACIDITIES

\author{
A Thesis \\ Presented to \\ The Faculty of the Department of Chemistry \\ San José State University \\ In Partial Fulfillment \\ of the Requirements for the Degree \\ Master of Science
}

by

Saul Perez

December 2015 
(C) 2015

Saul Perez

ALL RIGHTS RESERVED 
The Designated Thesis Committee Approves the Thesis Titled

\title{
FORMATION OF ORGANIC FILMS ON SULFURIC ACID SOLUTIONS AT UPPER TROPOSPHERE AND LOWER STRATOSPHERE AEROSOL ACIDITIES
}

\author{
by
}

Saul Perez

\begin{abstract}
APPROVED FOR THE DEPARTMENT OF CHEMISTRY
SAN JOSÉ STATE UNIVERSITY
\end{abstract}

December 2015

$\begin{array}{ll}\text { Dr. Annalise Van Wyngarden } & \text { Department of Chemistry } \\ \text { Dr. Roger Terrill } & \text { Department of Chemistry } \\ \text { Dr. J. R. David Brook } & \text { Department of Chemistry }\end{array}$ 


\begin{abstract}
FORMATION OF ORGANIC FILMS ON SULFURIC ACID SOLUTIONS AT UPPER TROPOSPHERE AND LOWER STRATOSPHERE AEROSOL ACIDITIES
\end{abstract}

\title{
By Saul Perez
}

The effects of atmospheric aerosols on climate remain uncertain, especially where aerosol chemical composition is not well known. Chemical analysis of aerosols in the upper troposphere (UT) and lower stratosphere (LS) show sulfuric acid (40 - 80 wt. \%) and water as the major components. However, recently, the presence of organic molecules was also reported in UT/LS aerosols which could produce a broad spectrum of chemical reactions that have the potential to alter the aerosol climate properties, so experiments were performed to examine these reactions. Propanal, individually or in mixtures with glyoxal and/or methylglyoxal, combined with sulfuric acid formed highly colored surface films. Since surface films would control aerosol climate properties, the goals of this work are to determine the chemical composition of the films and to determine the effects of aging, organic mixture, and acidity on film composition and formation rates. Films and solutions were analyzed by attenuated total reflectance-FTIR, ${ }^{1} \mathrm{H}$ NMR and UV-Vis spectroscopies. Results show that aldol condensation products (mainly 2-methyl-2-pentenal and 1,3,5-trimethylbenzene) and acetals (mainly 2,4,6triethyl-1,3,5-trioxane and longer-chain linear polyacetals) are the major species in films formed from propanal. In mixtures of multiple organics, cross-reactions between propanal and glyoxal and/or methylglyoxal were also observed. These results are used to assess the potential for organic films to affect the climate properties of UT/LS aerosols. 


\section{ACKNOWLEDGEMENTS}

I would like to thank my adviser Dr. Van Wyngarden for allowing me to work in this project and to successfully finish it. I have also words of gratitude to the members of my committee Dr. Terrill and Dr. Brook for their patience and support.

My special thanks to my friends and colleagues, Linda Leong, Kieu Ha, Nathan Feick, and Jeffrey Berry for their help, hard work and suggestions of new experiments.

I owe my deepest gratitude, and I will be forever in debt with Gustaf, Thomas, and all the Arrhenius's family because they show me that nothing is impossible and made me always feel at home.

My gratitude to my friend Julio Valdivia-Silva for his friendship and his constant support and encouragement in the difficult times. In the same way to my friend Lauren Fletcher.

I want to thank to my classmates and graduate student fellows, in special to my friends Jamie Lunkley, Victoria Chang, Chris Hoff, and John Kim.

I dedicate this thesis to my little daughter Ana, who was always waiting for me with a smile together with my beloved wife, Erika. 


\section{TABLE OF CONTENTS}

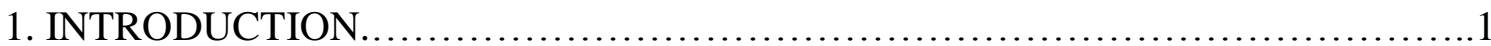

1.1. Aerosols in the Upper Troposphere and Lower Stratosphere and their Effects on

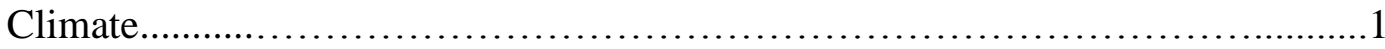

1.2. Potential Reactions of Organics in Sulfuric Acid Aerosols............................4

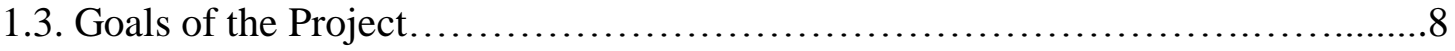

2. EXPERIMENTAL METHODS................................................ 10

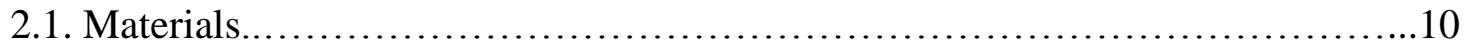

2.2. Film Formation Survey Experiments..........................................11

2.3. Chemical Analysis of Films..................................................12

2.3.1. Collection of the Film Samples..........................................12

2.3.2. Attenuated Total Reflectance (ATR)-FTIR Spectroscopy...............................13

2.3.3. Proton Nuclear Magnetic Resonance ( ${ }^{1} \mathrm{H}$ NMR) Spectroscopy...............15

2.3.4. Ultraviolet-visible Absorption Spectroscopy of Solutions.....................16

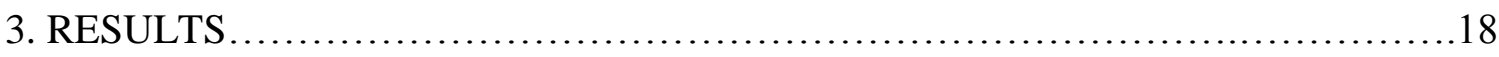

3.1. Organic Surface Film Formation............................................ 18

3.2. Chemical Composition of Surface Films.....................................20

3.2.1. Aldol Condensation Products...........................................21

3.2.2. Ethers: Acetals/Hemiacetals and Linear/Cyclic Polyacetals.................25

3.2.3. Other Potential Film Components: Organosulfates, etc.....................27

3.3. Effect of Acidity ...................................................................

3.4. Cross-Reactions with Glyoxal and Methylglyoxal.............................32 


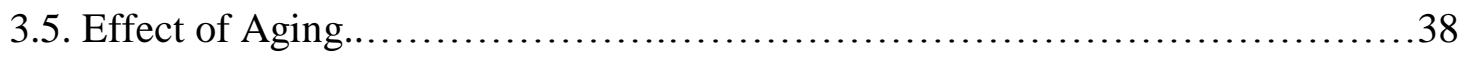

3.6. UV-visible Spectrometric Analysis of Solutions..............................42

4. CONCLUSIONS AND FURTHER STUDIES ................................46

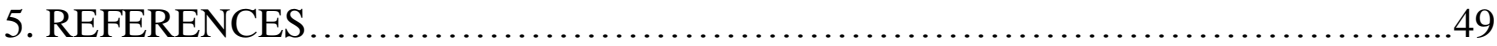

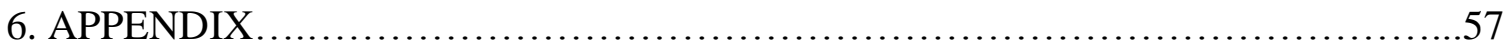




\section{TABLES}

Table 1: Sample sulfuric acid solution titrations with target concentrations shown in the top raw.................................................................

Table A1: FTIR results from surface films formed on 7-day-old mixtures of propanal $(\mathrm{P})$, glyoxal $(\mathrm{G})$ and/or methylglyoxal $(\mathrm{M})$ in 48 wt. $\% \mathrm{H}_{2} \mathrm{SO}_{4}$, compared with neat standards of 2-methyl-2-pentenal (2M2P), 1,3,5 trimethylbenzene (TMB), and $2,4,6$-triethyl-1,3,5-trioxane ................................................. 57

Table A2 : ${ }^{1} \mathrm{H}$ NMR results from surface films formed on mixtures of propanal (P), glyoxal (G) and/or methylglyoxal(M) compared with neat standards of 2-methyl-2pentenal (2M2P), 1,3,5 trimethylbenzene (TMB), 2,4,6-triethyl-1,3,5-trioxane (T)

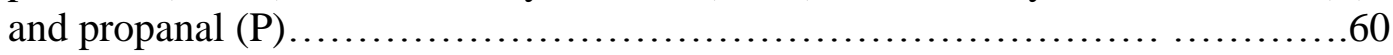




\section{LIST OF FIGURES}

Figure 1: Two-day-old film formed on $0.30 \mathrm{M}$ propanal in $48 \mathrm{wt} . \% \mathrm{H}_{2} \mathrm{SO}_{4} \ldots \ldots \ldots \ldots . .13$

Figure 2: Schematic diagram of ATR sampling accessory...........................15

Figure 3: Daily observations of film-formation rates from various organic/sulfuric acid mixtures stored in the dark at room temperature

Figure 4: ATR-FTIR spectra of a surface film (7 days old) formed on $0.30 \mathrm{M}$ propanal in 48 wt. $\% \mathrm{H}_{2} \mathrm{SO}_{4}$ (green) and of neat standards of propanal, 2-methyl-2-pentenal (2M2P), 1,3,5-trimethylbenzene (TMB) and 2,4,6-triethyl-1,3,5-trioxane (T) scaled to indicate their maximum possible contribution to the film...............22

Figure 5: ${ }^{1} \mathrm{H}$ NMR spectrum of a surface film (39 days old) formed on $0.30 \mathrm{M}$ propanal

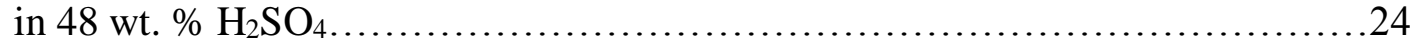

Figure 6: ATR-FTIR spectra of surface films (7 days old) formed on mixtures of $0.30 \mathrm{M}$ propanal $(\mathrm{P})$ in $7.02 \mathrm{M} \mathrm{H}_{2} \mathrm{SO}_{4}(48$ wt. \%) and $6.77 \mathrm{M} \mathrm{HCl} \ldots \ldots \ldots \ldots \ldots \ldots . . . . . .28$

Figure 7: ${ }^{1} \mathrm{H}$ NMR spectrum of a surface film (6 days old) formed from $0.30 \mathrm{M}$ propanal

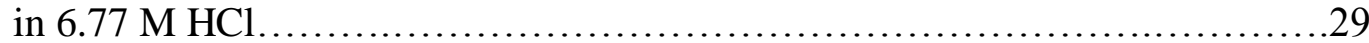

Figure 8: ATR-FTIR spectra of surface films (2, 3 and 33 days old) formed on mixtures

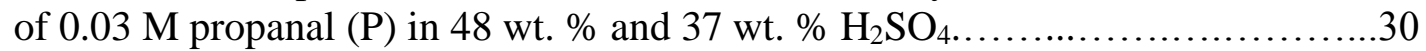

Figure 9: ${ }^{1} \mathrm{H}$ NMR spectrum of a surface film (36 days old) formed on $0.30 \mathrm{M}$ propanal

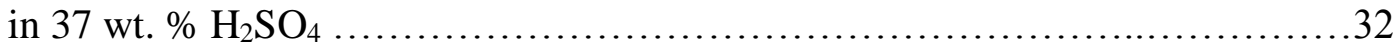

Figure 10: ATR-FTIR spectra of surface films (7 days old) formed on mixtures of propanal (P); propanal and glyoxal (PG); propanal and methylglyoxal (PM); and propanal, glyoxal and methylglyoxal (PGM) in 48 wt. \% $\mathrm{H}_{2} \mathrm{SO}_{4}$

Figure 11: ${ }^{1} \mathrm{H}$ NMR spectrum of a surface film (39 days old) formed on a mixture of

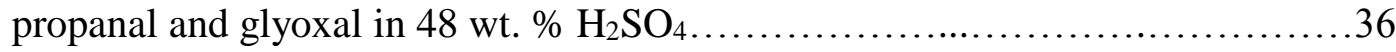

Figure 12: ${ }^{1} \mathrm{H}$ NMR spectrum of a surface film (39 days old) formed on a mixture of

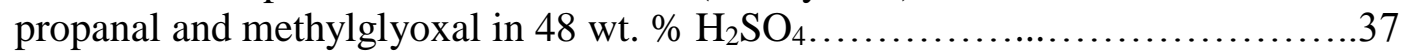

Figure 13: ${ }^{1} \mathrm{H}$ NMR spectrum of a surface film (6 days old) formed on a mixture of propanal, glyoxal and methylglyoxal in $48 \mathrm{wt} . \% \mathrm{H}_{2} \mathrm{SO}_{4} \ldots \ldots \ldots \ldots \ldots \ldots \ldots . . . \ldots 37$

Figure 14: Effect of aging on the ATR-FTIR spectra of a surface film $(2,7,33$, and 37 days old) formed on a mixture of $0.30 \mathrm{M}$ propanal (P) in $48 \mathrm{wt} \% \mathrm{H}_{2} \mathrm{SO}_{4} \ldots \ldots \ldots 40$ 
Figure 15: Effect of aging on the ATR-FTIR spectra of a surface film $(3,7,33$, and 37 days old) formed on a mixture of $0.30 \mathrm{M}$ propanal (P) in $37 \mathrm{wt} . \% \mathrm{H}_{2} \mathrm{SO}_{4} \ldots \ldots \ldots . .40$

Figure 16: Effect of aging on the ATR-FTIR spectra of a surface film $(2,7,33$, and 37 days old) formed on a mixture of propanal and glyoxal (PG) in 48 wt. \% $\mathrm{H}_{2} \mathrm{SO}_{4}$

Figure 17: Effect of aging on the ATR-FTIR spectra of a surface film $(2,7,33$, and 37 days old) formed on a mixture of propanal and methylglyoxal (PM) in $48 \mathrm{wt}$ \% $\mathrm{H}_{2} \mathrm{SO}_{4}$.

Figure 18a: UV-Vis absorption of 274-day-old organic solutions in 48 wt. $\% \mathrm{H}_{2} \mathrm{SO}_{4}$ after removal of film/precipitate..............................................43

Figure 18b: UV-Vis absorption of 274-day-old organic solutions in 76 wt. $\% \mathrm{H}_{2} \mathrm{SO}_{4}$ after removal of film/precipitate ................................................. 


\section{LIST OF ABBREVIATIONS}

1,3,5-trimethylbenzene

TMB

2,4.6-triethyl-1,3,5-trioxane

$\mathrm{T}$

2-methyl-2-pentenal

2M2P

Trimethylsilane

TMS

Amorphous material transmitting infrared radiation

AMTIR

Attenuated total reflectance

ATR

Cloud condensation nuclei

$\mathrm{CCN}$

Deuterated chloroform

$\mathrm{CDCl}_{3}$

Fourier transform infrared spectroscopy

FTIR

Glyoxal

G

High performance liquid chromatography

HPLC

Intergovernmental panel on climate change

IPCC

Internal reflection element

IRE

Methylglyoxal

M

Proton nuclear magnetic resonance

${ }^{1} \mathrm{H}$ NMR

Carbonyl sulfide

OCS

Propanal

$\mathrm{P}$

Tandem mass spectrometry

MS/MS

Ultraviolet-visible

UV-Vis

Upper troposphere/lower stratosphere

UT/LS 


\section{INTRODUCTION}

\subsection{Aerosols in the Upper Troposphere and Lower Stratosphere and Their Effects on Climate.}

An aerosol is a suspension of liquid or solid particles in a gas, with particles ranging in size from nanometers to tens of microns. ${ }^{1,2}$ Atmospheric aerosols have a wide variety of sources that include natural and anthropogenic origins. The effects of atmospheric aerosols on climate are complex and not completely understood. Over the last few decades, a variety of groups have investigated the influence of atmospheric aerosols. ${ }^{1,3-7}$ However, their effects remain the largest physical uncertainty in the prediction of climate change according to an assessment by the 2013 Intergovernmental Panel on Climate Change (IPCC). ${ }^{8}$

The effects of aerosols on climate may be direct or indirect, changing the balance of incoming solar radiation and outgoing terrestrial radiation in the atmosphere and resulting in warming or cooling. ${ }^{6,8}$ The direct aerosol effect is a result of scattering, reflection, and/or absorption of radiation, changing the net energy budget of the Earth-atmosphere system..$^{1,2,8,9}$ The indirect aerosol effect is related to the role of aerosol particles as cloud condensation nuclei $(\mathrm{CCN})$, which are the seeds for the formation of clouds. ${ }^{8}$ Changes in aerosol particle number, size, and/or chemical composition have the ability to affect climate by changing cloud lifetimes, reflectivities and precipitation patterns. Since the exact magnitude of these effects on climate caused by aerosols remains uncertain, it is important to gain insight into the optical (light scattering and absorption) and cloudforming properties of aerosols. ${ }^{1,2,9}$ These properties are, in turn, strongly influenced by 
aerosol chemical composition and, therefore, by chemical reactions that occur within aerosols. $^{8}$

The upper troposphere and lower stratosphere (UT/LS) region can range roughly from $\sim 8$ to $20 \mathrm{~km}^{10}$ in altitude and varies with geographical region and season since the altitude of the boundary between the UT and the LS (tropopause) depends on factors such as temperature, wind, and pressure. Thus, the tropopause varies from $\sim 10 \mathrm{~km}$ in the extratropical regions to $\sim 17 \mathrm{~km}$ in the tropics. ${ }^{10}$ The tropopause roughly corresponds to a minimum in the vertical temperature profile of the atmosphere, so the UT/LS region is characterized by very cold temperatures $\left(-73\right.$ to $\left.-53{ }^{\circ} \mathrm{C}\right) .{ }^{11-14}$ Therefore, most water vapor condenses and falls before reaching the UT/LS. This lack of water results in aerosols composed of aqueous sulfuric acid $\left(\mathrm{H}_{2} \mathrm{SO}_{4}\right)^{15-17}$ at high concentrations of 40 to 80 wt. $\%{ }^{18}$ This acidic profile is the result of the formation of $\mathrm{H}_{2} \mathrm{SO}_{4}$ from the reaction of $\mathrm{SO}_{2}$ with water. The major sources of $\mathrm{SO}_{2}$ are volcanic eruptions and the successive oxidation of carbonyl sulfide (OCS). ${ }^{16,19-22}$ The presence of sulfuric acid aerosols in the upper troposphere and in the lower stratosphere plays an important role in climate. Sulfuric acid aerosols are highly reflective to UV-visible radiation. Therefore, climate models assume that their main effect is to scatter incoming solar radiation, resulting in cooling at the surface of the Earth. ${ }^{23-25}$ However, recent airborne measurements showed that a significant fraction of upper troposphere and lower stratosphere (UT/LS) aerosols also contain organic material. ${ }^{7,26,27}$ The effects of this material on aerosol climate properties are highly uncertain due to its unknown chemical composition. ${ }^{8,26}$ A potential source of this organic material is the uptake of gas phase organic compounds into sulfuric acid aerosols, leading to reactions 
that could potentially produce high molecular weight products that remain in the aerosol phase. This organic material could alter the radiative and/or cloud forming properties of the UT/LS aerosols by causing more light absorption or by altering hygroscopicity (and thereby inhibiting water uptake), respectively. ${ }^{26,28}$

Herein, we examine the effects of reactions of sulfuric acid with organic molecules on the chemical composition of bulk laboratory solutions that model aerosols in the UT/LS. Results obtained from initial laboratory experiments carried out by our group in which various carbonyl species (propanal, glyoxal, and/or methylglyoxal) were combined with sulfuric acid at UT/LS aerosol acidities resulted in highly-colored solutions. Importantly, solutions containing propanal also demonstrated the formation of colored organic films which appeared on the surface of the aqueous reaction mixtures.

The formation of surface films is of particular interest, since surface coatings on aerosols could have dramatic effects on aerosols properties since they would be expected to control the hygroscopicity and the optical properties of aerosols and, therefore, could affect climate. Thus, this work focuses on identifying which reaction(s) of propanal in sulfuric acid solutions are responsible for the formation of surface films in order to assess whether these reactions would be expected to alter the climate properties of UT/LS aerosols. 


\subsection{Potential Reactions of Organics in Sulfuric Acid Aerosols.}

Low molecular weight carbonyl compounds taken up by aerosols have been shown to undergo various reactions capable of producing higher molecular weight species (accretion reactions) ${ }^{29-32}$ under tropospheric conditions (much less acidic that UT/LS aerosols). These reactions include aldol condensation, hemiacetal/acetal formation, further polymerization of acetals, cyclotrimerization, and organosulfate formation. ${ }^{33-36}$ Since all of these reactions are either acid-catalyzed or require sulfate, they are expected to be even more important in the sulfuric acid aerosols typical of the UT/LS. We consider each of these reactions described below and illustrated in Schemes 1 and 2 as potential candidates for producing high molecular weight products that are likely to partition into solid surface films. Scheme 1 shows the formation of diols and enols that can go on to participate in the accretion reactions shown in Scheme 2. First, aldehydes and ketones react with water to varying degrees to form $\operatorname{diols}^{35}$ (Scheme 1a) that provide the alcohol groups required for formation of acetals/hemiacetals and organosulfates (Scheme $2 b$ and f ${ }^{35}$. Second, carbonyl compounds in an acidic aqueous medium may also form enols $\mathrm{s}^{35,37}$ (Scheme 1b) that will allow the acid-catalyzed formation of aldol-condensation

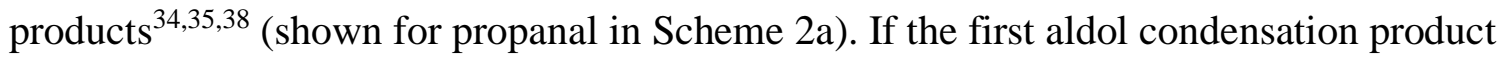
(2-methyl-2-pentenal in Scheme 2a) continues to react, it will add additional carbons to the chain or backbone, forming the linear trimer, 2,4-dimethyl-2,4-heptadienal (Scheme 2a) and potentially larger polymers (Scheme 2a) that could partition to the surface. These polymers have conjugation that increases with each aldol condensation step and, therefore, can produce significant UV-vis absorbance that shifts further into the visible 
with each step. Linear trimers produced by aldol condensation may also cyclize; for example, trimethylbenzene is formed via cyclization of the trimer of propanal, 2,4dimethyl-2,4-heptadienal ${ }^{39}$ (Scheme 2b). Alternatively, carbonyl species can undergo acid-catalyzed reactions with their hydrated forms (diols) (Scheme 1a) to form hemiacetals and acetals $\mathrm{s}^{35,37,40}$ as shown for propanal in Scheme 2c. If the reaction continues, it will form linear polyacetal products ${ }^{35,40}$ (Scheme 2d). Trioxane formation via cyclotrimerization is also possible and is shown for propanal in Scheme 2e. ${ }^{41,42}$ Finally, organosulfate formation can occur when diols formed from carbonyls (Scheme 1a) react directly with sulfuric acid to produce sulfate esters (Scheme $2 f) .43-46$

Products of propanal aldol condensation reactions have been reported in previous studies in sulfuric acid (60-96 wt. \%) solutions by Noziere and Esteve and Casale et. al. ${ }^{34}$; both studies focused on the potential ability to form light-absorbing compounds and, therefore, were not sensitive to any other non-absorbing reaction products of propanal. Linear products of further aldol condensation of propanal in aqueous media using zeolites as a catalyst have been reported to cyclize and form 1,3,5-trimethylbenzene (Scheme 2b).$^{39}$ The formation of acetal/hemiacetal and cyclic products (Scheme $2 \mathrm{c}-\mathrm{e}$ ) have not been reported for propanal in sulfuric acid, but they have been observed for other aldehydes as reported by Garland et al. ${ }^{40}$ and Li et al. ${ }^{42}$ Similarly, although organosulfates (Scheme 2f) have not been reported to be formed by propanal, they have been observed to form in reactions of glyoxal on sulfuric acid aerosols by Liggio et al.$^{45}$ and in reactions of various alcohols with sulfuric acid. ${ }^{44,46-51}$ 
Scheme 1: Enolization and Hydration of Carbonyl Groups.

1a) Hydration of ketones and aldehydes:<smiles>[CH+]C=C[PH2+]C=O</smiles>

1b) Enolization of ketones and aldehydes:<smiles>[R7]C([R])=C([R7])O</smiles>

Scheme 2: Potential Reactions of Propanal in the Presence of Sulfuric Acid.

2a) Aldol condensation:

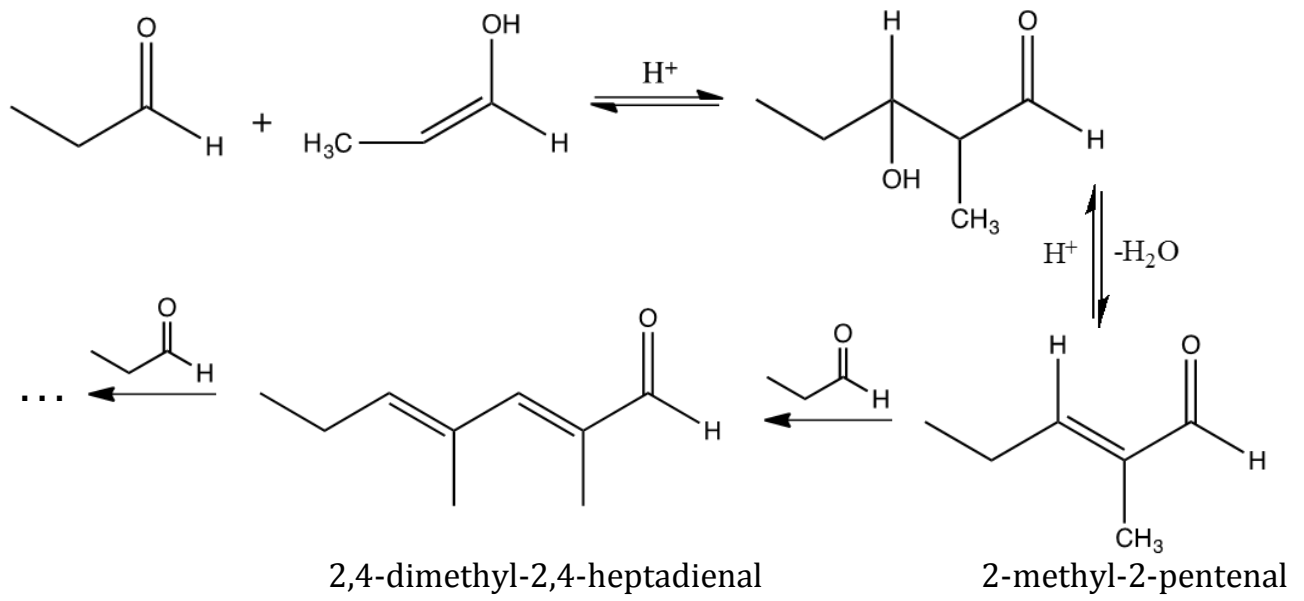


2b) Trimethylbenzene formation<smiles>C#CNC(=O)C(C)=CC(C)=CCC</smiles>

2,4-dimethyl-2,4-heptadienal

1,3,5-trimethylbenzene

2c) Hemiacetal and acetal formation

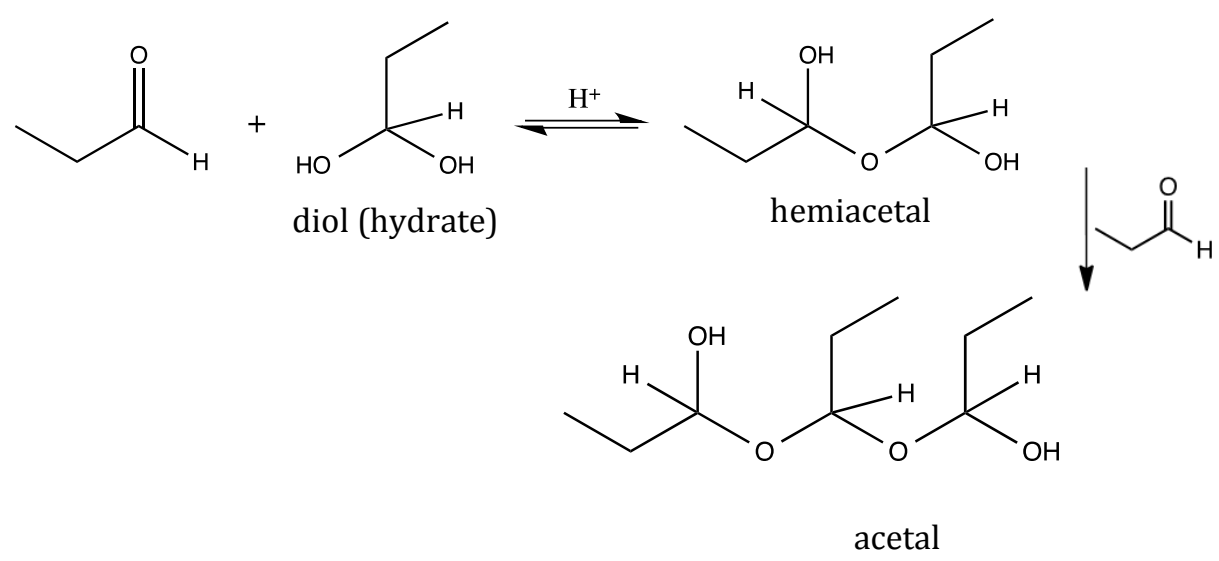

2d) Further polymerization

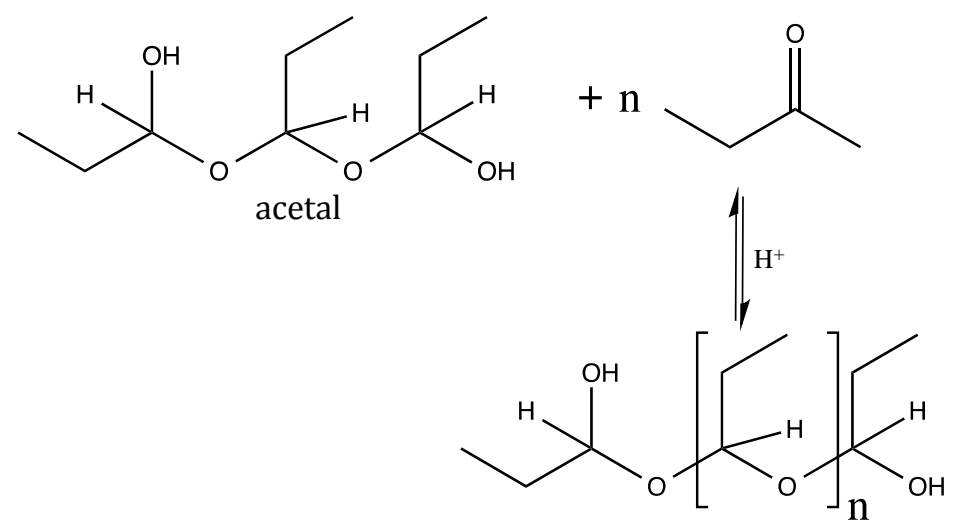

polyacetal 
2e) Trimerization of propanal

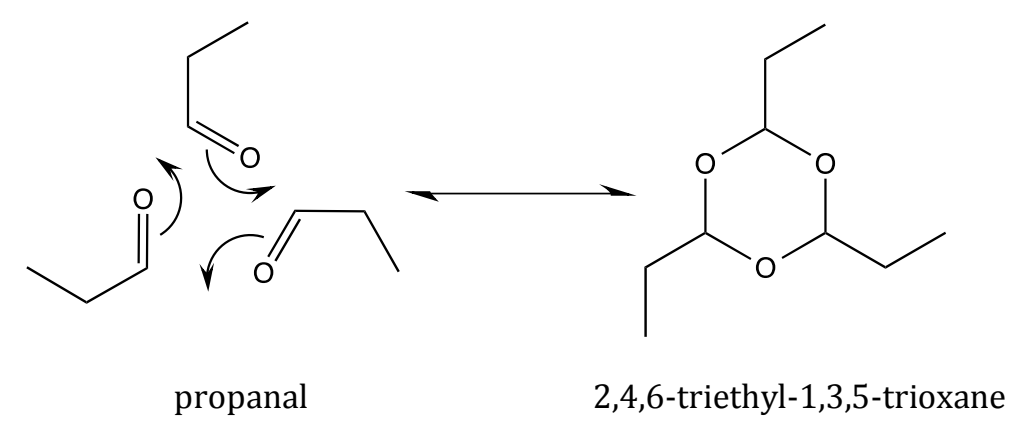

2f) Organosulfate formation

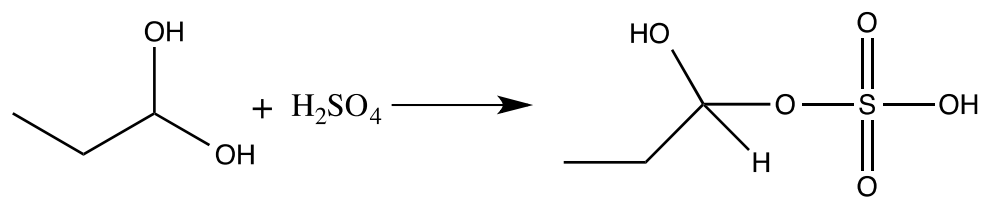

\subsection{Goals of the Project.}

The possible role of reactions of organic molecules with sulfuric acid in UT/LS aerosols and their implications for the climate properties of aerosols are examined. Due to the large number of potential organic products formed in UT/LS aerosols and the inaccessibility of the UT/LS without high altitude-aircraft, it is difficult to obtain detailed information about the chemical composition of mixed organic/sulfuric acid aerosols when formed in situ. Our laboratory experiments, although under simplified conditions, are designed to improve our understanding of the chemical process(es) responsible for organic film formation from reactions of propanal in sulfuric acid in order to assess the potential importance of similar processes in UT/LS aerosols.

The first aim of this study is to evaluate the conditions (acidity and organic mixture) under which surface films are formed by the reactions of small carbonyl species with 
sulfuric acid at acidities typical for aerosols in the UT/LS. ${ }^{26}$ The second and primary aim is to use Fourier transform infrared (FTIR), nuclear magnetic resonance (NMR) and UVvisible spectroscopies to analyze the chemical composition of surface films formed in the laboratory in order to determine which type(s) of reaction(s) are responsible for film formation. Identification of the film-forming reactions will enable us to assess their potential to impact climate properties of aerosols under more complex UT/LS conditions. 


\section{EXPERIMENTAL METHODS}

\subsection{Materials.}

The reagents used in this study were 40 wt. \% methylglyoxal (Sigma-Aldrich), 40 wt. \% glyoxal (Sigma-Aldrich), 97 wt. \% propanal (reagent grade, Sigma-Aldrich), 97 wt. \% 2-methyl-2-pentenal (Sigma-Aldrich), 96-98 wt. \% sulfuric acid (ACS reagent grade, Sigma-Aldrich), 37 wt. \% hydrochloric acid (ACS reagent grade, Sigma-Aldrich), deuterated chloroform (ultra-pure, Sigma-Aldrich), and 0.5 N sodium hydroxide standard solution (Sigma-Aldrich). Ultrapure water $(10$ to $16 \mathrm{M} \Omega \cdot \mathrm{cm})$ was used for preparing the diluted solutions.

Concentrated sulfuric acid (96-98 wt. \%) was diluted with water to obtain 19, 37, 48 and 76 wt. \% stock solutions. The stock sulfuric acid solutions were titrated in triplicate with $0.5 \mathrm{~N}$ sodium hydroxide standard solutions in order to confirm the acid concentrations. Typical results are shown in Table 1.

Table 1: Sample sulfuric acid solution $(5 \mathrm{ml})$ titrations with target concentrations shown in the top row.

\begin{tabular}{|c|c|c|c|c|c|c|c|}
\hline \multicolumn{2}{|c|}{$\begin{array}{c}76.0 \text { wt. } \% \mathrm{H}_{2} \mathrm{SO}_{4} \\
(13.0 \mathrm{M})\end{array}$} & \multicolumn{2}{c|}{$\begin{array}{c}48.5 \text { wt. } \% \mathrm{H}_{2} \mathrm{SO}_{4} \\
(6.69 \mathrm{M})\end{array}$} & \multicolumn{2}{c|}{$\begin{array}{r}36.5 \text { wt. \% } \mathrm{H}_{2} \mathrm{SO}_{4} \\
(4.72 \mathrm{M})\end{array}$} & \multicolumn{2}{c|}{$\begin{array}{c}19.4 \text { wt. \% } \mathrm{H}_{2} \mathrm{SO}_{4} \\
(2.24 \mathrm{M})\end{array}$} \\
\hline $\mathrm{NaOH} \mathrm{ml}$ & Molarity & $\mathrm{NaOH} \mathrm{ml}$ & Molarity & $\mathrm{NaOH} \mathrm{ml}$ & Molarity & NaOH ml & Molarity \\
\hline 53.00 & 13.05 & 27.20 & 6.70 & 19.30 & 4.75 & 9.16 & 2.26 \\
\hline 53.00 & 13.05 & 27.00 & 6.65 & 19.25 & 4.74 & 9.10 & 2.24 \\
\hline 51.80 & 12.76 & 27.10 & 6.67 & 19.25 & 4.74 & 9.16 & 2.26 \\
\hline & & & & 19.20 & 4.73 & & \\
\hline AVG & $\mathbf{1 2 . 9 5}$ & & $\mathbf{6 . 6 7}$ & & $\mathbf{4 . 7 4}$ & & $\mathbf{2 . 2 5}$ \\
\hline SD & 0.17 & & 0.02 & & 0.01 & & 0.01 \\
\hline
\end{tabular}




\subsection{Film Formation Survey Experiments.}

Preliminary experiments combining propanal $(\mathrm{P})$, and its mixtures with glyoxal $(\mathrm{G})$, and/or methylglyoxal (M) with sulfuric acid solutions showed that the surface films formed at rates which were affected by the concentration of acid, choice and concentration of organic species, and the light exposure. Therefore, controlled experiments were performed to determine which sample compositions and reaction conditions produced detectable surface films and the rates at which these organic films were produced.

In this work, solutions of propanal, propanal/glyoxal, propanal/methylglyoxal (1:1) and propanal/glyoxal/methylglyoxal (1:1:1) were prepared at $0.030 \mathrm{M}$ in each organic present. Each organic mixture was prepared at four aqueous sulfuric acid concentrations: $19,37,48$, and 76 wt. $\% \mathrm{H}_{2} \mathrm{SO}_{4}$. The concentration of $0.030 \mathrm{M}$ in each organic species was chosen to be on the upper end of the range of reasonable concentrations for carbonyl species in atmospheric aerosols, which vary dramatically with geographic location. Concentrations of propanal, glyoxal, and methylglyoxal have not been directly measured in UT/LS aerosols; however, concentrations of glyoxal and methylglyoxal in lower tropospheric aerosols have been estimated to be in the millimolar range from measurements of mass loadings of specific organic species combined with typical total aerosol mass loadings. ${ }^{52-58}$

After thorough mixing in volumetric flasks, samples of $4.0 \mathrm{ml}$ of each reaction mixture were pipetted into $8 \mathrm{ml}$ glass vials and sealed with Teflon-lined caps and parafilm. Samples were stored in the dark at $21-24^{\circ} \mathrm{C}$ (room temperature). Samples were 
observed daily over a period of 180 days in order to determinate whether or not surface films were present and how quickly they formed. The observations of film formation were made visually by tilting the vials gently to facilitate the detection of solid films as evidenced by their resistance to movement or tendency to form flakes. This procedure was performed gently in order to minimize disturbance of the surface films.

\subsection{Chemical Analysis of Films.}

\subsubsection{Collection of the Film Samples.}

The films formed from solutions that were $0.030 \mathrm{M}$ in the organic reagent presented the difficulty that the amount of film produced was very small. Under these conditions, solid film material could not be removed reliably without contamination by sulfuric acid which interferes with spectroscopic analysis of the films. Therefore, the concentrations of the organic reagents were increased ten-fold to $0.30 \mathrm{M}$ to produce more film, which facilitated the collection of film without disturbing the underlying sulfuric acid. Volumetric flasks $(100 \mathrm{ml})$ were used to store the solutions in order to concentrate the film on the small surface area in the neck of the flask as shown in Figure 1. Once filled with solution, each volumetric flask was closed using a glass stopper and additionally sealed with parafilm. As the acid-catalyzed reaction of organic compounds proceeded, a thin, dark organic layer formed on the surface of the solutions (Figure 1). This solid or semi-solid material was collected for subsequent analysis using a clean glass rod for FTIR or the tip of a Pasteur pipette for NMR. A portion of the outer surface film was removed and carefully transferred onto the surface of an attenuated total reflectance (ATR) crystal for ATR-FTIR or transferred into $\mathrm{CDCl}_{3}$ solvent in an NMR tube. 
During the process of collecting the samples, efforts were made to exclusively collect the organic film while minimizing the amount of aqueous phase by avoiding contact of the glass rod or Pasteur pipette with the underlying aqueous solution. Aqueous phase exclusion is important as water and sulfuric acid interfere with the spectral elucidation of the organic functional groups of interest.

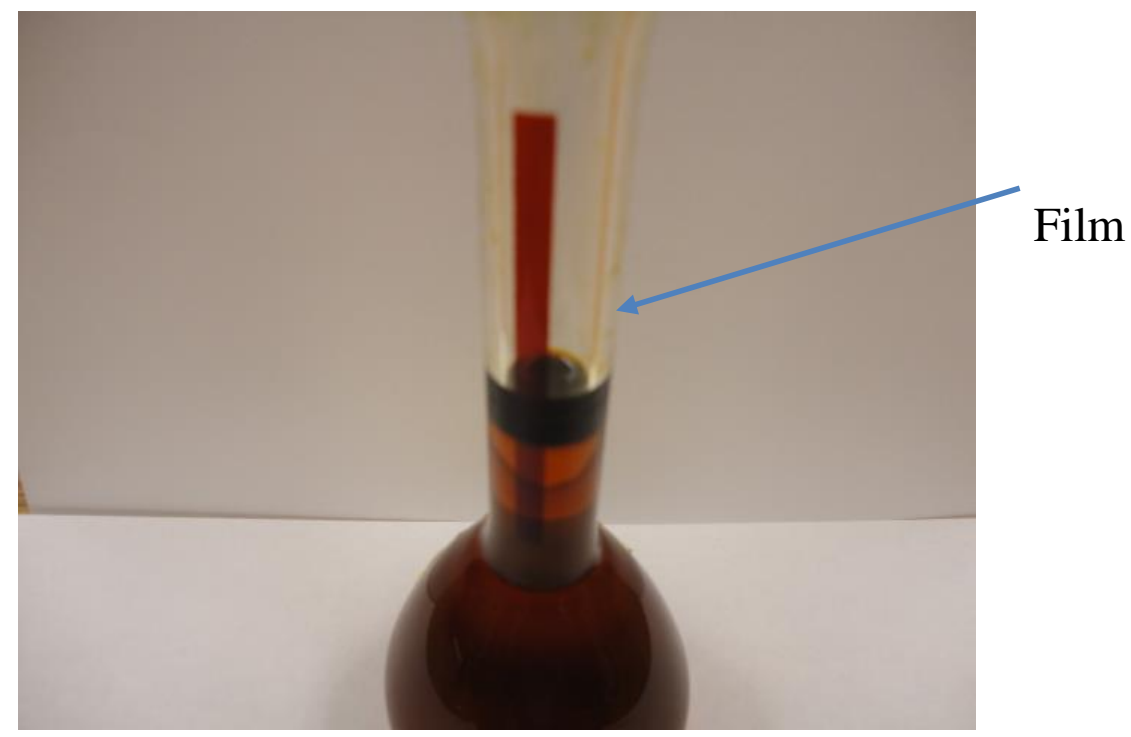

Figure 1: Two-day-old film formed on $0.30 \mathrm{M}$ propanal in 48 wt. $\% \mathrm{H}_{2} \mathrm{SO}_{4}$

\subsubsection{Attenuated Total Reflectance (ATR)-FTIR Spectroscopy.}

Film samples were analyzed by attenuated total reflectance (ATR)-FTIR spectroscopy which was chosen since it does not require alteration of the chemical environment by dissolution in a solvent and because it can be used with highly acidic samples. ${ }^{59-62}$ ATR is a type of internal reflection spectroscopy in which the sample is placed in contact with an internal reflection element (IRE) of high refractive index. Infrared radiation is focused onto the edge of the IRE, reflected through the IRE, and then directed to the detector, as shown in Figure $2 .{ }^{60-62}$ When a sample is present, it absorbs 
some of the IR radiation, allowing measurement of the IR absorption spectrum of the sample. ATR combined with FTIR spectroscopy allows for the convenient examination of solid and liquid samples that can be placed directly on the ATR crystal (often at the bottom of a shallow trough). In particular, for our samples, ATR also avoids traditional salt plates that would be dissolved by any sulfuric acid.

The FTIR instrument used was a Nicolet 6700 spectrophotometer with single IR beam and a Michelson interferometer. The FTIR beam was produced by a thermal EverGlo source and split by a KrB beam splitter, and a mercury cadmium telluride (MCT) detector was used. All spectra were collected under the following conditions: spectral range of $650-4000 \mathrm{~cm}^{-1}, 32$ scans, $1 \mathrm{~cm}^{-1}$ resolution, and a screen to attenuate the IR beam to $20 \%$ for the highly sensitive MCT detector. A routine check of the beam intensity and spectrum was performed before every experiment. After this quick inspection, a background spectrum was collected with the clean ATR crystal.

An AMTIR crystal consisting of highly homogeneous $\mathrm{As}_{\mathrm{x}} \mathrm{Se}_{\mathrm{y}} \mathrm{Ge}_{\mathrm{z}}$, was chosen as the IRE since it is insoluble in water, and compatible with concentrated acid. The crystal is fixed with an angle of incidence of 45 degrees, and 10 total reflections and has a refractive index of $2.5 .^{63}$ The crystal is mounted on an optical bench that allows precise, repeatable positioning of the crystal. The films were transferred directly from the volumetric flask to the surface of the crystal with the help of a glass rod and carefully distributed on the surface, avoiding direct contact of the glass rod with the surface to prevent scratching. After each film was deposited on the surface of the ATR crystal, a 
room temperature FTIR spectrum was collected. Between samples, the crystal surface was cleaned with acetone and then put through a blank run to check for contamination.

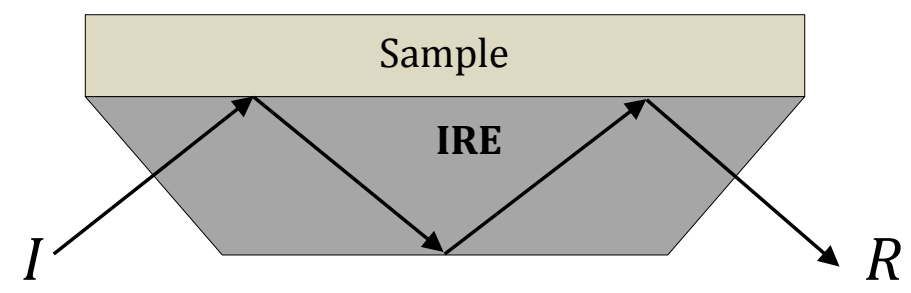

Figure 2: Schematic diagram of ATR sampling accessory (I=Incident radiation; R= Reflected radiation, IRE Internal Reflection Element)

\subsubsection{Proton Nuclear Magnetic Resonance ( ${ }^{1} \mathrm{H}$ NMR) spectroscopy.}

Proton NMR spectroscopy $\left({ }^{1} \mathrm{H}\right.$ resonance) was used to obtain further information about the composition of the film. ${ }^{1} \mathrm{H}$ NMR experiments were performed at ambient temperature using a Varian INOVA-400 spectrometer. Tetramethylsilane (TMS) was used as the internal standard for calibrating chemical shift. Quartz NMR tubes (5 mm outer diameter) were used to contain the samples and deuterated chloroform $\left(\mathrm{CDCl}_{3}\right)$ was used to dissolve the samples. Using a Pasteur pipette, a small drop of the sample with an approximate mass of $4 \mathrm{mg}$ was transferred directly to an NMR tube previously filled with $\mathrm{CDCl}_{3}$ to a height of 3.8 to $5.1 \mathrm{~cm}$ (around $0.6 \mathrm{ml}$ ). The tube was covered with a polyethylene cap and then agitated briefly to dissolve the sample. 


\subsection{Ultraviolet-visible Absorption Spectroscopy of Solutions.}

Solutions of the organic/ $\mathrm{H}_{2} \mathrm{SO}_{4}$ mixtures were analyzed by $\mathrm{UV}$-vis spectroscopy to evaluate the presence of chromophores present in the aqueous acidic solution that might also be involved in formation of the solid and semisolid films. The solutions presented coloration that indicated the probable presence of aldol condensation products.

Quantitative analysis using ultraviolet-visible (UV-vis) spectroscopy is based on the light absorption capacity of molecules and atoms that can be expressed in terms of the transmittance $(\mathrm{T})$ or absorbance $(\mathrm{A})$ of a solution contained in a transparent cell having a specific path length (b). The relationship between absorbance and the concentration (c) of an absorbing analyte is represented by Beers Law:

$$
\text { Equation } 1 \quad A=-\log T=\log \frac{P o}{P}=\epsilon b c
$$

where $\epsilon$ is the molar absorption or extinction coefficient and $P_{o}$ and $P$ are the incident and transmitted radiation intensities, respectively. ${ }^{61}$ The effective molar absorptivity was calculated based on the concentration of propanal only in order to easily evaluate the effects of adding glyoxal and methylglyoxal to propanal/sulfuric acid mixtures.

The UV-visible spectra of the solutions were obtained using a Varian Cary 50 Bio UV-visible spectrophotometer model with a diode array detector at a resolution of 0.6 $\mathrm{nm}$. The light source was a xenon flash lamp with a spectral range of 190 to $800 \mathrm{~nm}$. A Peltier unit with circulating water bath at $23{ }^{\circ} \mathrm{C}$ was used to control temperature. Sample solutions were placed in quartz cuvettes of 0.001 to $1 \mathrm{~cm}$ of length for analysis. 
Reference spectra were measured in sulfuric acid solutions at the same $\mathrm{H}_{2} \mathrm{SO}_{4}$ concentration as the sample. Samples were prepared at $0.030 \mathrm{M}$ in each organic (as in section 2.2) and were filtered to eliminate solid residue from broken film, using $2.5 \mu \mathrm{m}$ Teflon filters and a glass syringe with a Teflon O-ring compatible with concentrated sulfuric acid. The filtered solution was transferred into the quartz cuvettes using a glass Pasteur pipette. Between samples, concentrated sulfuric acid was used to remove possible organic molecules attached to the wall of the cuvettes, followed by a copious amount of ultrapure water to remove the remaining sulfuric acid. 


\section{RESULTS}

Section 3.1. describes the formation of colored solutions, precipitates, and surface films from organic molecules combined with sulfuric acid and examines the impact of acidity and organic mixture on the film formation kinetics. Section 3.2. presents results

from chemical analysis of surface films by FTIR and ${ }^{1} \mathrm{H}$ NMR spectroscopy. The impacts of different conditions on film composition are evaluated by changing the acidity, composition of the organic mixture, and age in Sections 3.3, 3.4 and 3.5, respectively. Section 3.6. describes UV-vis spectra of organic/ $\mathrm{H}_{2} \mathrm{SO}_{4}$ solutions. The reactions principally responsible for the formation of films are identified by combining FTIR, ${ }^{1} \mathrm{H}$ NMR, and UV-vis results.

\subsection{Organic Surface Film Formation.}

Formation of colored solutions, precipitates and solid or semisolid films was observed in our preliminary experiments. ${ }^{64,65}$ In these experiments, a variety of carbonylcontaining organic compounds (propanal, glyoxal, and/or methylglyoxal) were mixed individually or in combination (0.030 $\mathrm{M}$ in each organic) with solutions of sulfuric acid (19-76 wt. \%), and then observed daily for the presence of film. Many propanalcontaining mixtures formed surface films on timescales from seconds to weeks after mixing. Mixtures containing only glyoxal and/or methylglyoxal did not form films.

In Figure 3, each of the four panels corresponds to one organic mixture (P, PG, PM or PGM) and presents results from daily film observations for each of the four acidities (76, 48, 37 and 19 wt. $\left.\% \mathrm{H}_{2} \mathrm{SO}_{4}\right)$ as a function of time. White indicates days that film was not observed and red indicates days that film was observed so that the formation of a film 
is represented by the beginning of a red bar. Interestingly, cycles of physical disintegration and re-formation of film (indicated by the end of a red bar and later beginning of a new red bar) were noticed in nearly all the films studied, with the exception of the propanal/glyoxal film formed on $37 \mathrm{wt} . \%$ acid. The results show that carbonyl compounds under acidic conditions may undergo reactions that result in the formation of surface films and that the rate of formation of films varies from seconds to months depending on the composition of the organic mixture and the acidity.

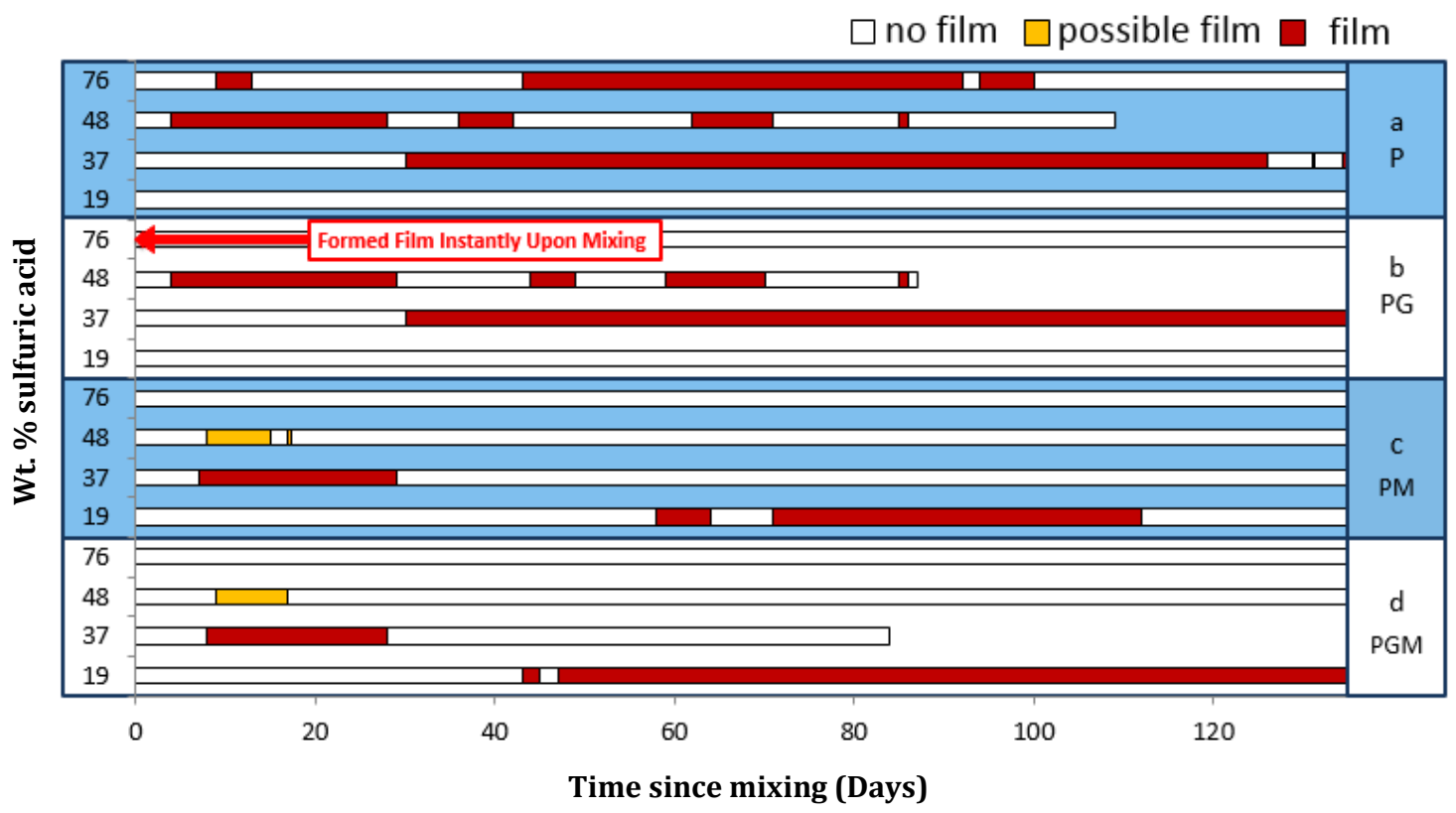

Figure 3: Daily observations of film formation rates from various organic/sulfuric acid mixtures stored in the dark at room temperature. Each blue or white panel corresponds to a different organic mixture: a) propanal (P); b) propanal and glyoxal (PG); c) propanal and methylglyoxal (PM), and d) propanal, glyoxal and methylglyoxal (PGM). Each organic is present at $0.030 \mathrm{M}$ and each panel shows solutions at the four acidities of 19, 37,48 and 76 wt. $\% \mathrm{H}_{2} \mathrm{SO}_{4}$. The horizontal bar for each solution is color-coded to indicate each day's observation: White indicates the absence of film, yellow indicates possible (uncertain) formation of film, and red indicates positive observation of film. A change of color in a bar indicates that an event has occurred, such as the formation or disintegration of a film indicated by the beginning or end of a red bar, respectively. 
The results in Figure 3 show that, overall, films form faster when the acidity of the solutions is increased. In fact, instant film formation was observed at the highest acidity when 76 wt. \% sulfuric acid was mixed with propanal and glyoxal. These results are consistent with the role of sulfuric acid as a catalyst $\mathrm{t}^{34,35,66}$ during film-forming reactions. Interestingly, with the exception of the propanal/glyoxal mixture, solutions of $76 \mathrm{wt} . \%$ $\mathrm{H}_{2} \mathrm{SO}_{4}$ do not form films or form them more slowly than at 48 wt. $\% \mathrm{H}_{2} \mathrm{SO}_{4}$. The reason may be that the low activity of the water in such concentrated sulfuric acid solutions prohibits reactions that require water such as aldol condensation and acetal formation.

With regard to the effect of organic mixture, the results from Figure 3 can be divided into two groups within which mixtures display similar film formation/ disintegration behavior, rates of film formation, and trends with acidity: One includes propanal and propanal/glyoxal films (Figure $3 a$ and $b$ ), and the second includes propanal/methylglyoxal and propanal/glyoxal/methylglyoxal films (Figure $3 \mathrm{c}$ and d). The difference between the two groups is the presence of methylglyoxal in the second group which shows that methylglyoxal changes the rates of formation of films and the trends with acidity in a complex manner. Specifically, in contrast to propanal and propanal/glyoxal, organic mixtures that included methylglyoxal at the highest concentration of sulfuric acid ( $76 \mathrm{wt} . \%)$ did not form films. This may indicate that methylglyoxal participates in cross-reactions with propanal resulting in products that do not partition to a surface film. Conversely, at the lowest acidity, formation of films only occurred when methylglyoxal was present in the organic mixture. 
Overall, these film formation survey experiments indicate that propanal and mixtures of propanal with glyoxal and/or methylglyoxal are able to form surface films when combined with sulfuric acid at acidities typical of upper troposphere/lower stratosphere aerosols. The complexity of the trends with acidity and organic mixture underscore the need to determine which reaction(s) are responsible for film formation.

\subsection{Chemical Composition of Surface Films.}

In this section, the chemical composition of films is analyzed by ATR-FTIR and NMR techniques. Films formed on $0.30 \mathrm{M}$ propanal in 48 wt. $\% \mathrm{H}_{2} \mathrm{SO}_{4}$ are used for this analysis as a starting point since surface films only formed when propanal was present and since propanal formed films fastest at 48 wt. $\% \mathrm{H}_{2} \mathrm{SO}_{4}$. Sections 3.3-3.5 subsequently address the effects of acidity, organic mixture and aging on the films. All the FTIR spectra of surface films shown in this section were scaled to the carbonyl peak at 1690 $\mathrm{cm}^{-1}$ corresponding to aldol condensation products (mainly 2-methyl-2pentenal) for comparison. Before scaling, a constant baseline was subtracted using the region from $4000-3950 \mathrm{~cm}^{-1}$ where the spectra were flat and the noise from water vapor was minimal. This scaling was necessary to allow comparisons among spectra of different samples because absolute absorbance intensity depends on the extent of coverage of the ATR crystal by the sample which could not be precisely controlled.

\subsubsection{Aldol Condensation Products.}

In this section, ATR-FTIR (Figure 4) ${ }^{67}$ and ${ }^{1} \mathrm{H}$ NMR (Figure 5) spectra of surface films formed on solutions of $0.30 \mathrm{M}$ propanal in $48 \mathrm{wt} . \% \mathrm{H}_{2} \mathrm{SO}_{4}$ are presented and used to analyze the chemical composition of the films. The results will help to understand 
which reactions are responsible for formation of films on organic/sulfuric acid solutions.

In Figure 4, ATR-FTIR spectra of the following neat standards are also presented for comparison: propanal, 2-methyl-2-pentenal (2M2P), 1,3,5-trimethylbenzene (TMB), and 2,4,6-triethyl-1,3,5-trioxane (T).

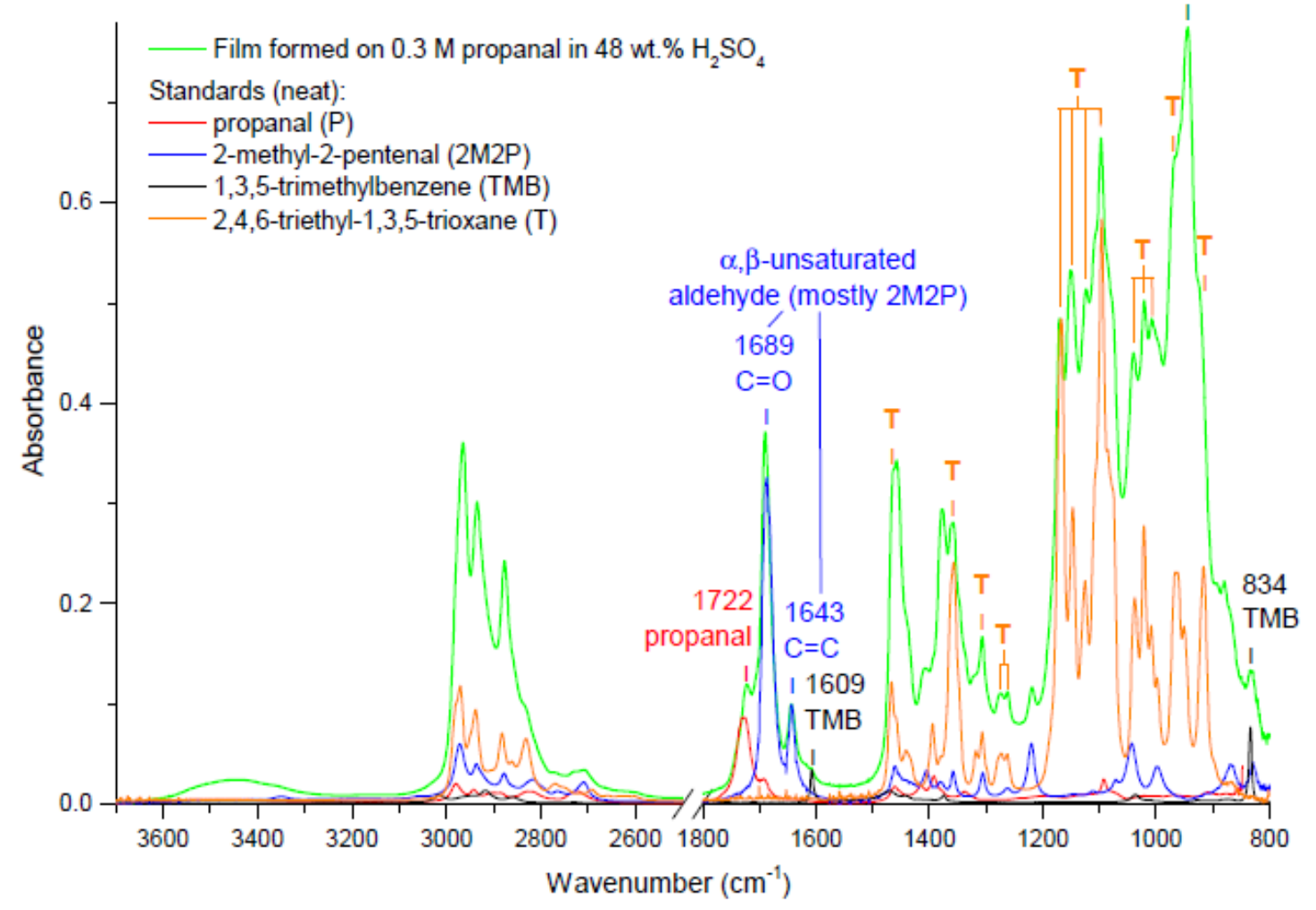

Figure 4: ATR-FTIR spectra of a surface film (7 days old) formed on $0.30 \mathrm{M}$ propanal in 48 wt. $\% \mathrm{H}_{2} \mathrm{SO}_{4}$ (green) and of neat standards of propanal, 2-methyl-2-pentenal (2M2P), 1,3,5-trimethylbenzene (TMB) and 2,4,6-triethyl-1,3,5-trioxane (T) scaled to indicate their maximum possible contribution to the film. The section from 2500 to $1800 \mathrm{~cm}^{-1}$ is omitted for clarity. The main peaks of the film are also tabulated in the Appendix, Table A1. (Reprinted with permission from Van Wyngarden et al., Atmos. Chem. Phys., $2015 .{ }^{67}$ )

The FTIR spectrum of the film (7 days old) in Figure 4 (green) indicates that aldolcondensation products are present in the film due to the presence of a strong $\mathrm{C}=\mathrm{O}$ peak at 
$1689 \mathrm{~cm}^{-1}$ in the region associated with $\alpha, \beta$-unsaturated aldehydes and the presence of the peak at $1643 \mathrm{~cm}^{-1}$ for the carbon-carbon double bond vibration. The first product of aldol condensation of propanal, 2-methyl-2-pentenal (2M2P) shown in Scheme 2a, is commercially available (Sigma-Aldrich) and its spectrum is scaled to indicate its maximum possible contribution to the spectrum of the film material produced from propanal/sulfuric acid in Figure 4. A second $\mathrm{C}=\mathrm{O}$ peak is present at $1721 \mathrm{~cm}^{-1}$ which falls in the region for saturated aldehydes and is assigned to unreacted propanal in the film.

The ${ }^{1} \mathrm{H}$ NMR spectrum in Figure 5 and peak position data from Table A2 in the Appendix also show evidence of aldol-condensation products in a 39-day-old film also formed on a $0.30 \mathrm{M}$ propanal, 48 wt. $\% \mathrm{H}_{2} \mathrm{SO}_{4}$ solution. Specifically, 2-methyl-2pentenal (2M2P) is identified as the dominant species by the following 5 strong peaks: The aldehyde group is confirmed by a strong singlet at $9.39 \mathrm{ppm}$. The vinyl proton is indicated by a triplet at $6.48 \mathrm{ppm}$. A quintuplet produced by splitting of the $\mathrm{CH}_{2}$ protons in the $\mathrm{CH}_{3}-\mathrm{CH}_{2}-\mathrm{CH}$ system appears at $2.38 \mathrm{ppm}$. The allylic methyl protons, are evident from a strong singlet at $1.74 \mathrm{ppm}$. The remaining methyl group is identified by a triplet at $1.11 \mathrm{ppm}$. A comparison with the spectrum of $2 \mathrm{M} 2 \mathrm{P}$ (our standard measurement) confirm these assignments. Overall, the identification of $2 \mathrm{M} 2 \mathrm{P}$ as the dominant aldol condensation product in the NMR spectrum allows assignment of the major $\mathrm{C}=\mathrm{O}\left(1689 \mathrm{~cm}^{-1}\right)$ peak in the FTIR spectrum (Figure 4) to 2M2P predominantly.

The ${ }^{1} \mathrm{H}$ NMR spectrum in Figure 5 also shows peaks at 2.28 and $6.79 \mathrm{ppm}$ that are assigned to 1,3,5-trimethylbenzene (TMB) by comparison to the literature spectrum $(\mathrm{SDBS})^{68}$. TMB formation was observed by Hoang et al. ${ }^{39}$ when propanal was allowed to 
react over acidic zeolite catalysts. They proposed that TMB was formed through cyclization and subsequent dehydration of the trimer (2,4-dimethyl-2,4-heptadienal) formed by aldol condensation of propanal (see Scheme 2b). In Figure 4, the FTIR spectrum of TMB (black) was included for comparison, demonstrating that the most intense bands in the TMB spectrum ( 834 and $1609 \mathrm{~cm}^{-1}$ ) are both observed in the spectrum of the film.

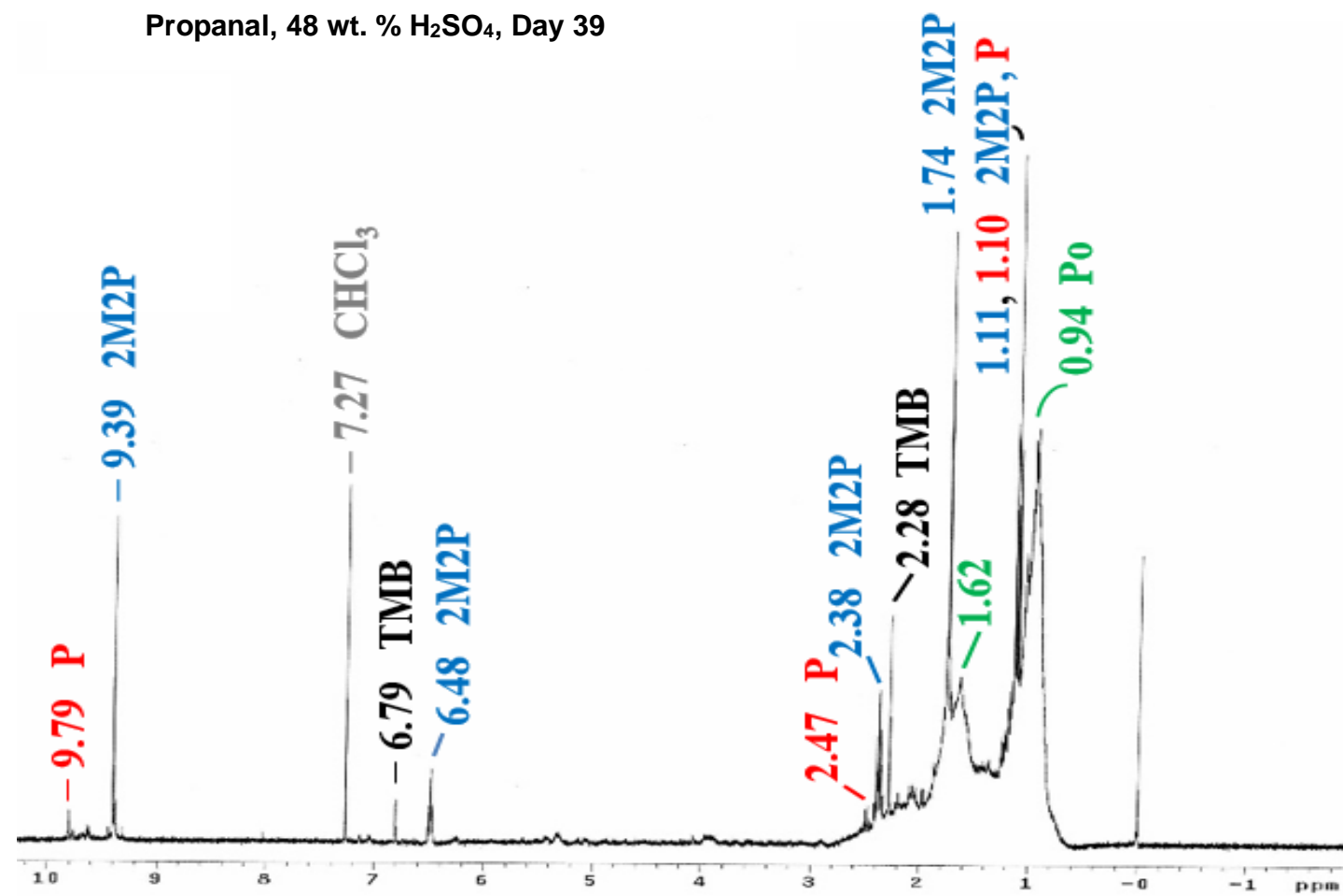

Chemical Shift (ppm)

Figure 5: ${ }^{1} \mathrm{H}$ NMR spectrum of a surface film (39 days old) formed on $0.30 \mathrm{M}$ propanal in 48 wt. $\% \mathrm{H}_{2} \mathrm{SO}_{4}$ and subsequently dissolved in $\mathrm{CDCl}_{3}$. Assignments to the following species are indicated: propanal (P), 2-methyl-2-pentenal (2M2P), 1,3,5-trimethylbenzene (TMB) and "polymer" (Po). 
Together, the FTIR and NMR results show that 2-methyl-2-pentenal (2M2P) and 1,3,5-trimethylbenzene (TMB) are the major products resulting from aldol reactions. However, the presence of many other strong peaks in the FTIR spectrum between 3000 $2800 \mathrm{~cm}^{-1}$ and $1500-800 \mathrm{~cm}^{-1}$ indicates a more complex chemical composition of the film, potentially including acetals and/or organosulfates.

\subsubsection{Ethers: Acetals/Hemiacetals and Linear/Cyclic Polyacetals.}

In addition to aldol condensation products, the FTIR spectrum also displays evidence for ether groups (C-O-C) due to strong peaks in the $1200-1000 \mathrm{~cm}^{-1}$ region (Figure 4). Species that could be responsible for these ether signatures include hemiacetals, acetals and/or higher order polyacetal polymers which can form from the reaction of propanal with one or more of its hydrates (diols) (Scheme 2c and 2d) or from cyclotrimerization of propanal to form the cyclic acetal, 2,4,6-triethyl-1,3,5-trioxane (T) (Scheme 2e). The presence of no polar groups could be responsible for the separation of the films from the aqueous medium.

Interestingly, the FTIR spectrum of the film confirms the presence of 2,4,6-triethyl1,3,5-trioxane (T) since at least 13 bands in the FTIR spectrum correspond to peaks in the spectrum of neat 2,4,6-triethyl-1,3,5-trioxane (Figure 4), including all six of the strongest peaks in the $1500-900 \mathrm{~cm}^{-1}$ region (see peaks labelled with an orange "T" in Figure 4). However, the reference NMR spectrum (SDBS ${ }^{68}$ for the trioxane contains peaks for a triplet at $4.78 \mathrm{ppm}$, a complex multiplet at $1.67 \mathrm{ppm}$ and a triplet at $0.94 \mathrm{ppm}$, which were not present in our NMR spectrum (Figure 5). The absence of the trioxane may be due to the age of this sample ( 39 days old vs. 7 days old for the FTIR spectrum), since 
our group recently identified its presence in the NMR spectra of younger films ( 7 days old $)^{67}$. Preliminary results from our laboratory also indicate the absence of $\mathrm{T}$ in the FTIR spectra of older samples in agreement with the results presented in this study. One possible explanation is that the trioxane and propanal are in "rapid" equilibrium, and the trioxane is depleted as propanal continues to react slowly over time via aldol condensation and/or linear acetal formation.

After assignment of FTIR peaks to 2-methyl-2-pentenal, trimethylbenzene, trioxane and propanal, the largest peak $\left(945 \mathrm{~cm}^{-1}\right)$ remains unassigned (Figure 4$)$. Therefore, the potential presence of hemiacetals and acetals (Scheme 2c) was evaluated. Hemiacetals specifically present bands in the $1150-1085 \mathrm{~cm}^{-1}$ region, while the acetal contains the CO-C-O-C moiety which would produce 5 characteristic bands between $1200-1020 \mathrm{~cm}^{-1}, 69$ so neither can account for the peak at $945 \mathrm{~cm}^{-1}$. Furthermore, both the hemiacetal and acetal are not major constituents since only a weak peak exists in the $\mathrm{OH}$ stretching region $\left(3500-3400 \mathrm{~cm}^{-1}\right.$ ) where a stronger peak (with respect to the peaks in the ether region) would be expected due to the $\mathrm{OH}$ groups.

Alternatively, the presence of longer-chain polymers from sequential reactions of propanal (Scheme 2d) potentially could explain the band at $945 \mathrm{~cm}^{-1}$ since stretching vibrations for longer-chain polymers with $-\mathrm{C}-\mathrm{O}-\mathrm{C}-\mathrm{O}-\mathrm{C}$ - backbones are shifted to lower frequency with the addition of multiple ether groups. For example, long-chain polymers of small molecules of aldehydes such as formaldehyde, acetal and propanal present broad and strong overlapping peaks in the $975-925 \mathrm{~cm}^{-1}$ region. ${ }^{70-74}$ Although the peak at 945 $\mathrm{cm}^{-1}$ does not exactly match the major peaks at 975,960 and $925 \mathrm{~cm}^{-1}$ in the Novak and 
Whalley ${ }^{70}$ spectrum of the polymethoxy polymer formed by pressurization of propanal, such broad absorption is observed in the $980-920 \mathrm{~cm}^{-1}$ region that a peak near $945 \mathrm{~cm}^{-1}$ may be obscured by overlapping peaks. Furthermore, the intensities of peaks from various polymer compounds in the film are likely to be different from those measured by Novak and Whalley due to a different degree of polymerization and/or different relative quantities of rotational isomers. ${ }^{70}$ The effect of the interaction between different species in the film matrix could also cause shifts in the positions of the polymer absorption bands. Although the ${ }^{1} \mathrm{H}$ NMR spectrum of the 39-day-old film (Figure 5), does not show peaks in the 4.5-5.0 ppm region that are characteristic for protons on the backbone of polymethoxy (-C-O-) n polymers ${ }^{73}$, our group ${ }^{67}$ has observed the presence of peaks in this region in the ${ }^{1} \mathrm{H}$ NMR spectra of samples that are the same age (7 days old) as the films used for FTIR in this study. It is possible that the older film has a greater variety of polymer lengths and structures so that individual peaks could just be weak and spread out. Additionally, both the 7- and 39-day-old films display a group of peaks between 11.7 ppm that are likely due to $\mathrm{CH}_{2}$ protons from long-chain polymers, and peaks near $0.94 \mathrm{ppm}$ that are likely due to $\mathrm{CH}_{3}$ groups from the same polymers. Finally, all major peaks in both the FTIR and the NMR spectra have been assigned or tentatively assigned to aldol condensation products, trioxane, long-chain polyacetals, or unreacted propanal.

\subsubsection{Other Potential Film Components: Organosulfates, etc.}

In this section, the potential presence of organosulfates and other species in the films is evaluated. 


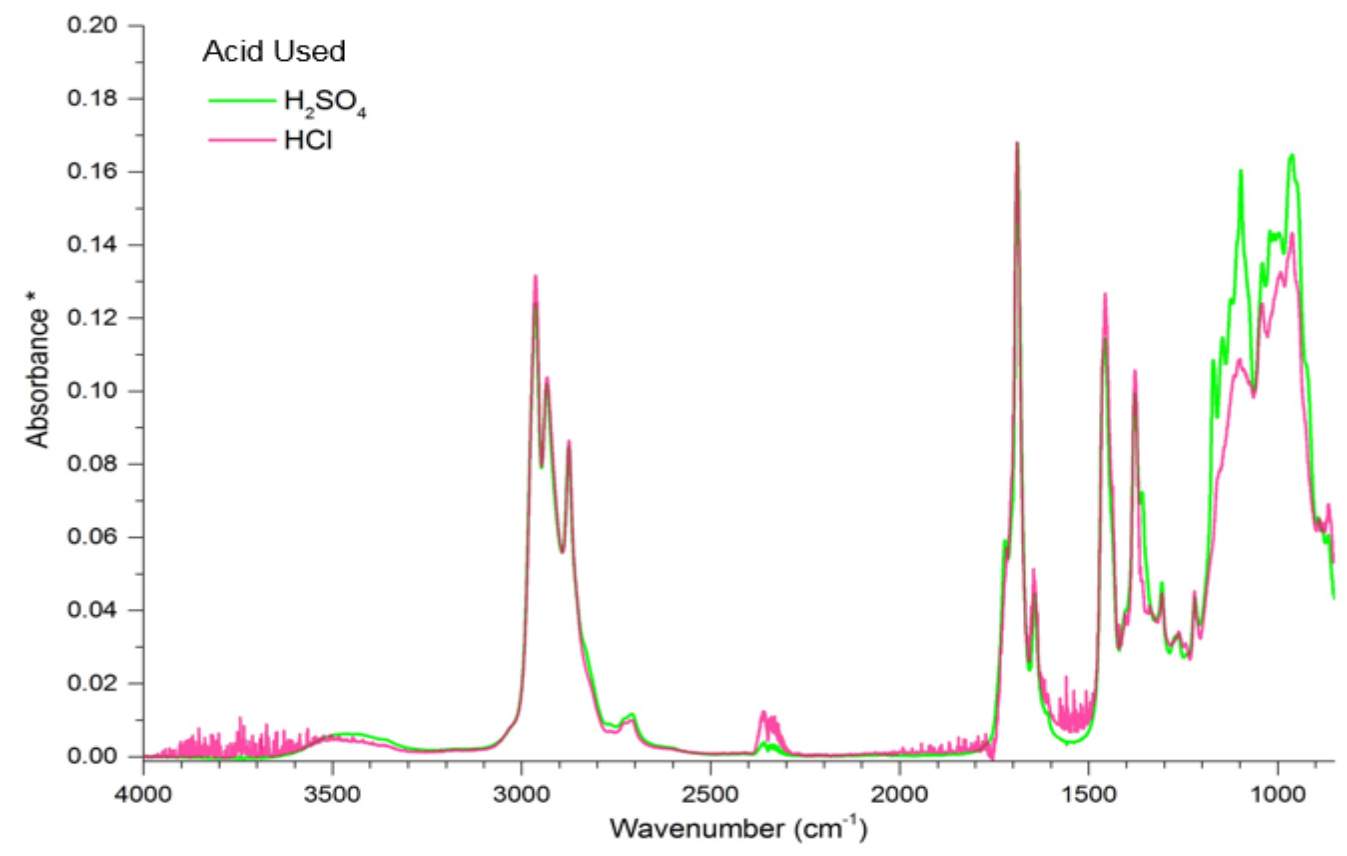

Figure 6: ATR-FTIR spectra of surface films (7 days old) formed on mixtures of $0.30 \mathrm{M}$ propanal (P) in $7.02 \mathrm{M} \mathrm{H}_{2} \mathrm{SO}_{4}(48$ wt. \%) and $6.77 \mathrm{M} \mathrm{HCl}$. The spectra were scaled to the carbonyl peak at $1690 \mathrm{~cm}^{-1}$.

In order to test for the presence of organosulfates in the films, solutions of $0.30 \mathrm{M}$ propanal were prepared in both 7.02 M sulfuric acid (48 wt. \%) and 6.77 M hydrochloric acid with similar $\mathrm{pH}$ values of -0.84 and -0.83 , respectively. Surface films formed on the $\mathrm{HCl}$ solution indicating that organosulfates are not necessary for the formation of films.

Figure 6 shows that the FTIR spectra of the films formed on both the sulfuric acid and the hydrochloric acid solutions are highly similar, demonstrating that organosulfates are not present in significant quantities. In agreement with the FTIR results, the ${ }^{1} \mathrm{H}$ NMR spectrum of the surface film made on the $\mathrm{HCl}$ solution (Figure 7) also lacks organosulfate peaks in the region of their resonance reported in the literature (10-12 ppm and around $3.0 \mathrm{ppm}$ for the proton of the sulfonic acid group). ${ }^{75,76,77}$ This is expected since, due to the 
ionizability/polarity of organosulfates, they would be expected to remain in the solution instead of partitioning to the film.

Propanal, 6.77 M HCl, Day 6

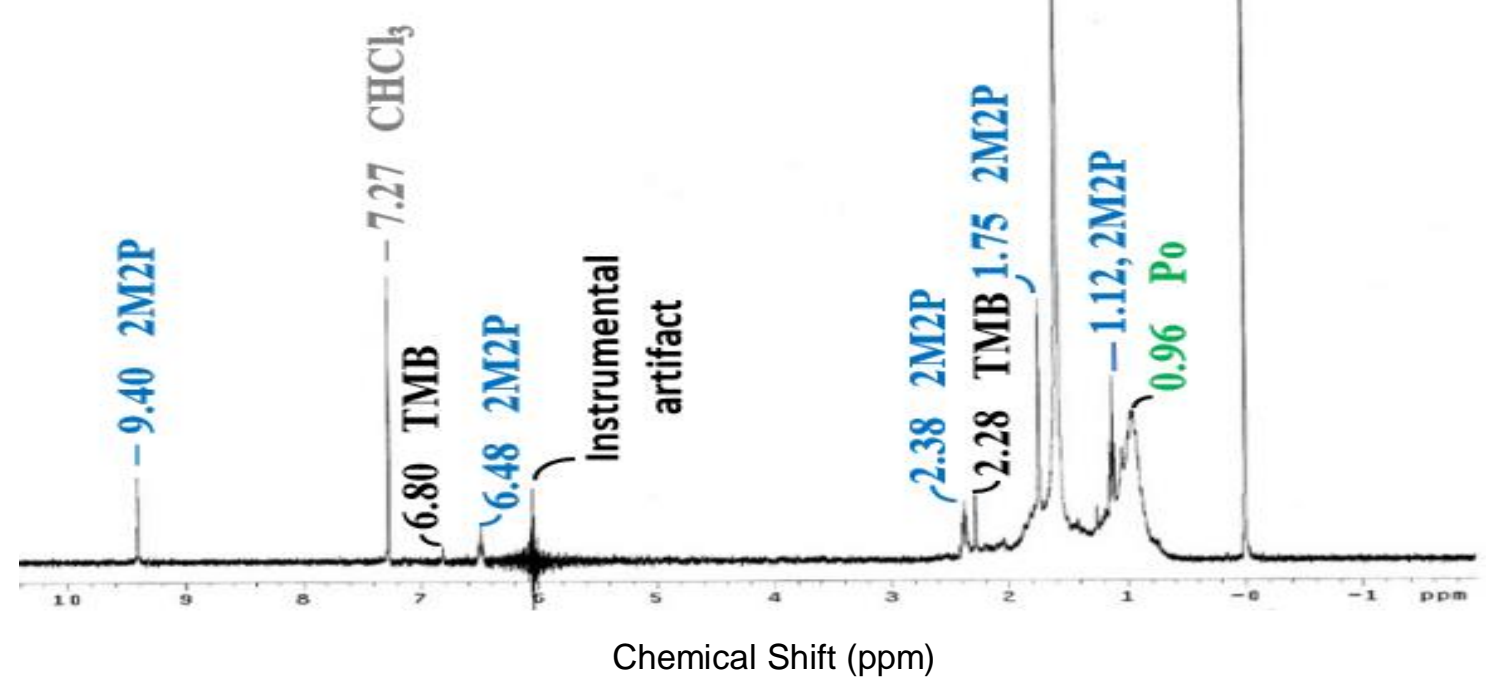

Figure 7: ${ }^{1} \mathrm{H}$ NMR spectrum of a surface film (6 days old) formed from $0.30 \mathrm{M}$ propanal in $6.77 \mathrm{M} \mathrm{HCl}$ and subsequently dissolved in $\mathrm{CDCl}_{3}$. Assignments to the following species are indicated: 2-methyl-2-pentenal (2M2P), 1,3,5-trimethylbenzene (TMB), and "polymer" (Po).

Although all major peaks in the FTIR and ${ }^{1} \mathrm{H}$ NMR spectra of the films formed on $0.30 \mathrm{M}$ propanal/48 wt.\% $\mathrm{H}_{2} \mathrm{SO}_{4}$ (Figures 4 and 5) have been assigned to aldol condensation products and acetals, some small, unassigned peaks remain, which indicate that other minor species are present in the film (e.g., ${ }^{1} \mathrm{H}$ NMR peaks between 1.8-2.2 and near $5.3 \mathrm{ppm}$ in Figure 5). These peaks could potentially be due to products of multiple aldol condensation steps, aldols that have not gone through the condensation process, the hemiacetal and acetal formed by propanal, other acetals that could also be formed by reactions of aldol condensation products with propanal, and/or products from oxidation of films by air/light. 


\subsection{Effect of Acidity.}

In Section 3.1, the effect of acidity on the rate of formation of films was discussed. The data collected from the film formation experiments using different organic molecules combined with 19,37 , and 48 wt. $\% \mathrm{H}_{2} \mathrm{SO}_{4}$ solutions indicate that films form faster at higher acidities. Interestingly, however, film formation rates at the highest acidity of 76 wt. $\% \mathrm{H}_{2} \mathrm{SO}_{4}$ were more complicated, with one mixture (PG) forming films instantly and the others (P, PM and PGM) forming films more slowly or not at all. In this section, the influence of acidity on the chemical composition of films formed by propanal is presented using two different concentrations of sulfuric acid: 37 wt. \% and 48 wt. \%.

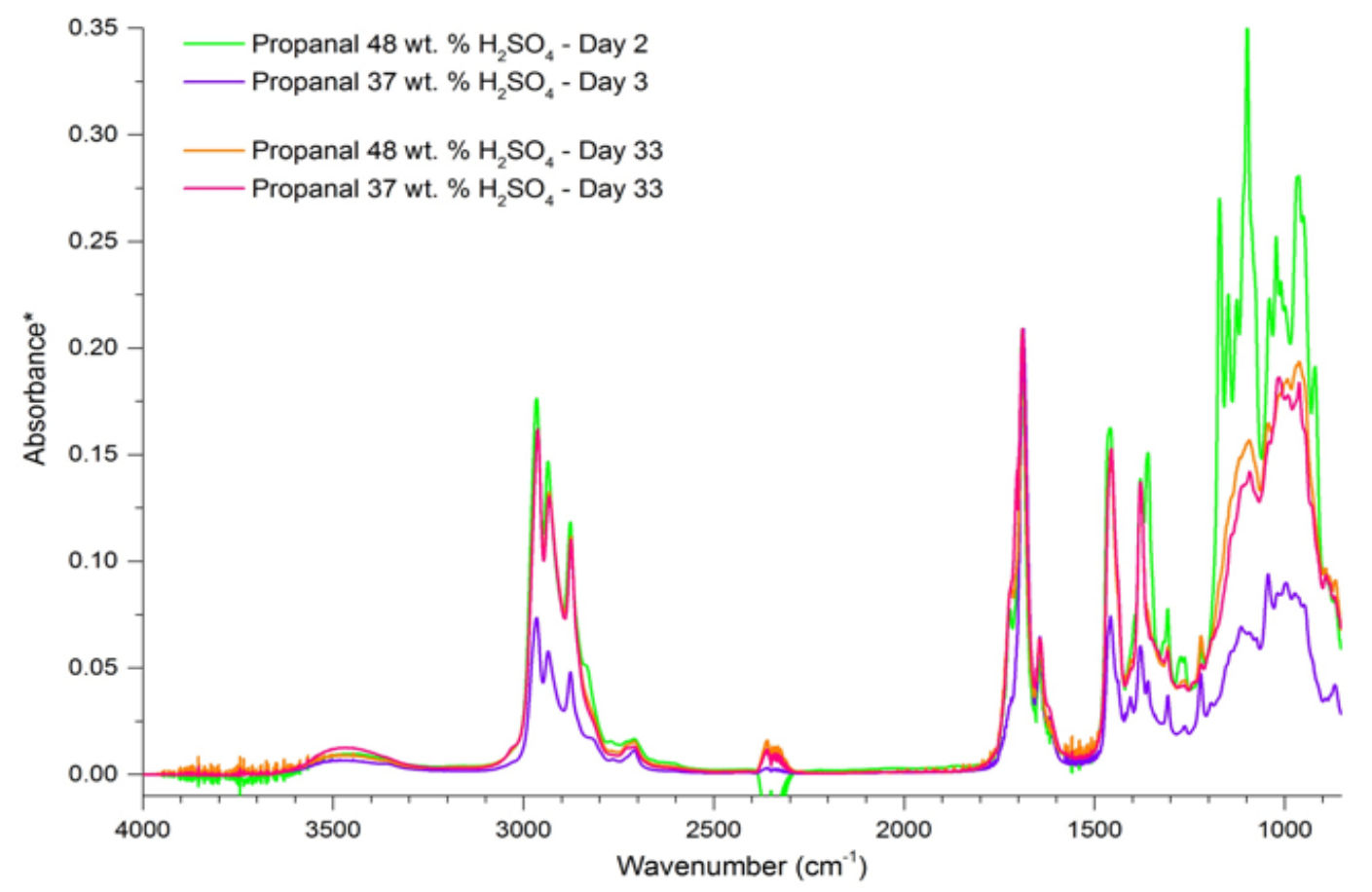

Figure 8: ATR-FTIR spectra of surface films (2,3 and 33 days old) formed on mixtures of $0.30 \mathrm{M}$ propanal (P) in $48 \mathrm{wt}$ \% and $37 \mathrm{wt} \% \mathrm{H}_{2} \mathrm{SO}_{4}$. All film spectra were scaled to the carbonyl peak at $1690 \mathrm{~cm}^{-1}$. 
In Figure 8, the spectra of surface films made from $0.30 \mathrm{M}$ propanal in $37 \mathrm{wt} \%$ and 48 wt. $\% \mathrm{H}_{2} \mathrm{SO}_{4}$ solutions are presented for films at two different ages: 2-3 days old and 33 days old. The FTIR spectrum of the young (2 day-old) film at 48 wt. $\% \mathrm{H}_{2} \mathrm{SO}_{4}$ (green line) presents strong bands for 2,4,6-triethyl-1,3,5-trioxane (T) in the 1200 - 900 $\mathrm{cm}^{-1}$ region as described in detail in Section 3.2.2 (Figure 4). Conversely, in the spectrum for the young (3-day-old) film at $37 \mathrm{wt} . \% \mathrm{H}_{2} \mathrm{SO}_{4}$, the peaks for $\mathrm{T}$ disappear or become weaker relative to the $\mathrm{C}=\mathrm{O}$ peak at $1689 \mathrm{~cm}^{-1}$. It is possible that the bands of $\mathrm{T}$ are overlapped by the presence of more hemiacetals, acetals, or polymers with long chains and/or that there is simply less trioxane in the lower acidity film. Interestingly, the 33day-old film spectra at both acidities appear very similar and do not present the strong bands for $\mathrm{T}$, indicating that the long-term effect of acidity on chemical composition may be negligible.

Figures 5 and 9 show the ${ }^{1} \mathrm{H}$ NMR spectra of 36- to 39-day-old propanal films formed on 48 and 37 wt. $\% \mathrm{H}_{2} \mathrm{SO}_{4}$ solutions, respectively. 2-methyl-2-pentenal (2M2P) and 1,3,5 trimethylbenzene (TMB) are positively identified at both acidities and $2 \mathrm{M} 2 \mathrm{P}$ is the dominant species in both cases. The polymethoxy polymer (Po) observed at $48 \mathrm{wt}$ \% $\mathrm{H}_{2} \mathrm{SO}_{4}$ also seems to be present at 37 wt. $\% \mathrm{H}_{2} \mathrm{SO}_{4}$ with peaks at $0.94,1.69$ and $4.98 \mathrm{ppm}$ in agreement with the FTIR results. 2,4,6-triethyl-1,3,5-trioxane (T) is missing from the ${ }^{1} \mathrm{H}$ NMR spectra at both acidities, also consistent with the lack of trioxane in the older FTIR spectra. Overall, our results show that films generally form faster at higher acidities and display significant chemical differences at young ages (2-3 days old), but quite similar spectra after aging for 36-39 days. 
Propanal, 37 wt. $\% \mathrm{H}_{2} \mathrm{SO}_{4}$, Day 36

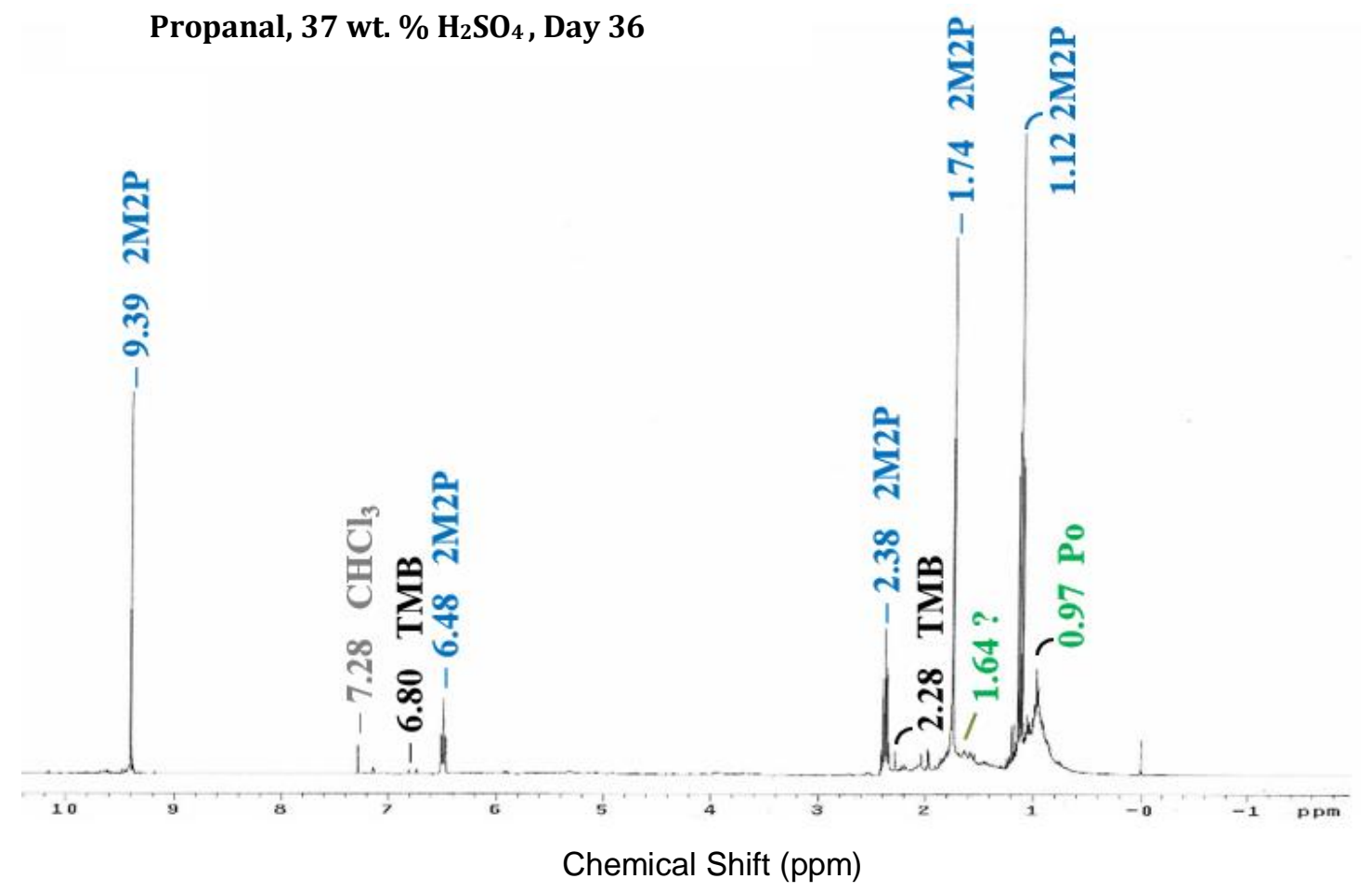

Figure 9: ${ }^{1} \mathrm{H}$ NMR spectrum of a surface film (36 days old) formed on $0.30 \mathrm{M}$ propanal in 37 wt. $\% \mathrm{H}_{2} \mathrm{SO}_{4}$ and subsequently dissolved in $\mathrm{CDCl}_{3}$. Assignments to the following species are indicated: 2-methyl-2-pentenal (2M2P), 1,3,5-trimethylbenzene (TMB), and "polymer" (Po).

\subsection{Cross-Reactions with Glyoxal and Methylglyoxal.}

In this section, the potential effect of the presence of additional organic species with carbonyl groups on the formation of films from propanal is evaluated. Comparisons of FTIR and ${ }^{1} \mathrm{H}$ NMR spectra of organic mixtures of propanal, propanal/glyoxal, propanal/methylglyoxal and propanal/glyoxal/methylglyoxal show the effect of organic mixture and provide evidence for cross-reactions between different organic species as follows. 


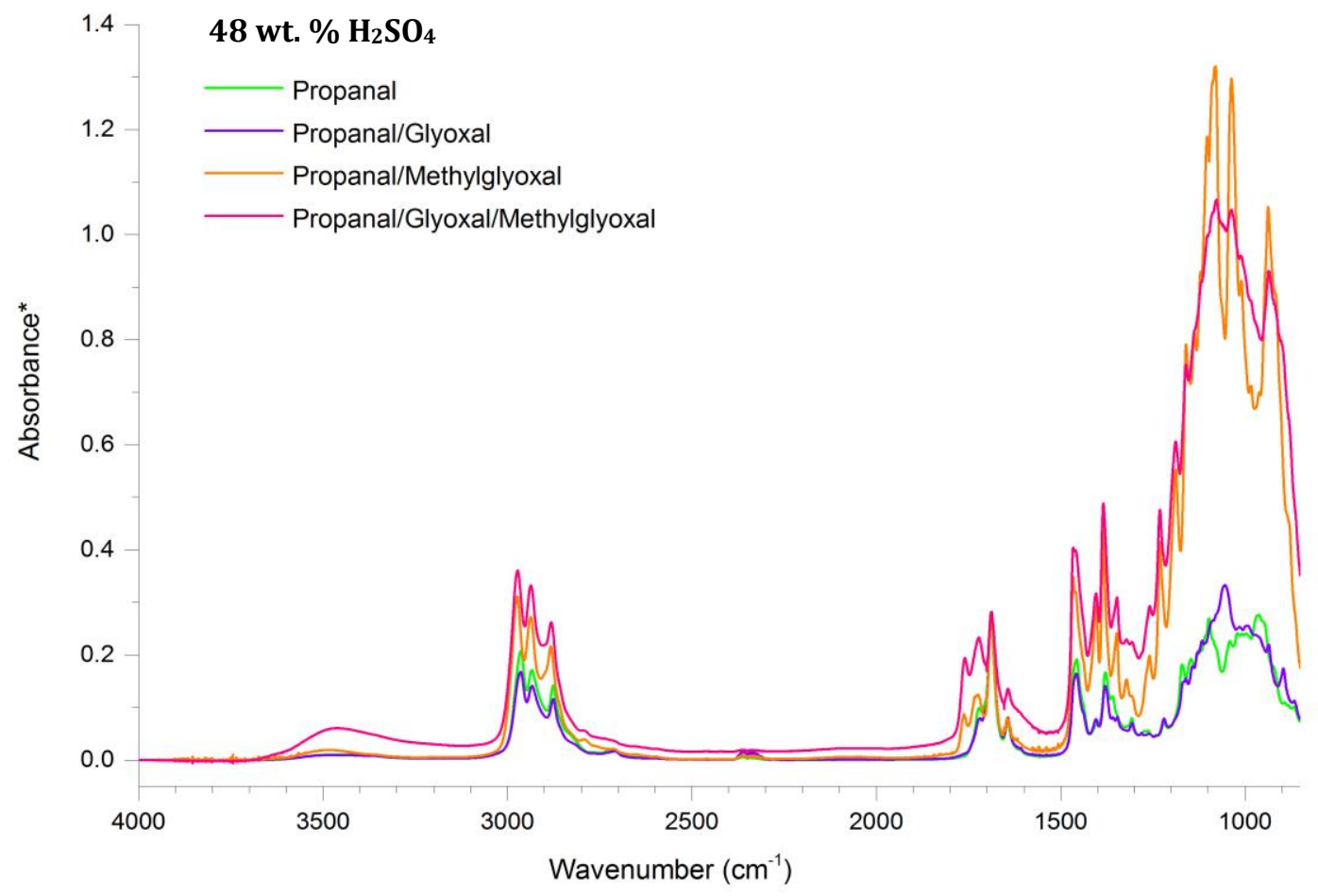

Figure 10: ATR-FTIR spectra of surface films (7 days old) formed on mixtures of propanal (P); propanal and glyoxal (PG); propanal and methylglyoxal (PM); and propanal, glyoxal and methylglyoxal (PGM) in 48 wt. $\% \mathrm{H}_{2} \mathrm{SO}_{4}$. Solutions are $0.30 \mathrm{M}$ in each organic. All film spectra were scaled to the carbonyl peak at $1690 \mathrm{~cm}^{-1}$.

Figure 10 shows films formed on mixtures of propanal (P), propanal/glyoxal (PG), propanal/methylglyoxal (PM) and propanal/glyoxal/methylglyoxal (PGM) in 48 wt. \% $\mathrm{H}_{2} \mathrm{SO}_{4}$ at $0.30 \mathrm{M}$ in each organic analyzed by ATR-FTIR 7 days after mixing. All the film spectra were scaled to the carbonyl peak at $1690 \mathrm{~cm}^{-1}$ to facilitate comparisons among the spectra. The spectra of the propanal and propanal/glyoxal films are very similar in the region from $1800-1600 \mathrm{~cm}^{-1}$ that corresponds to 2-methyl-2-pentenal and unreacted propanal. However, in the $1200-900 \mathrm{~cm}^{-1}$ region, the spectra for propanal and propanal/glyoxal differ from each other since the propanal/glyoxal spectrum does not match the spectral fingerprint for 2,4,6-triethyl-1,3,5-trioxane (T) like the spectrum of 
propanal-only does. This result suggests that glyoxal participates in cross-reactions with propanal, producing polyacetal polymers that absorb in the $1200-900 \mathrm{~cm}^{-1}$ region and contribute to the films.

The ATR-FTIR spectra (Figure 10) also show that when methylglyoxal is part of the initial organic mixture (propanal/methylglyoxal and propanal/glyoxal/methylglyoxal), there is a strong increase of the peaks in the acetal region $\left(1200-900 \mathrm{~cm}^{-1}\right)$ relative to the carbonyl peak at $1690 \mathrm{~cm}^{-1}$ indicating a larger acetal/aldol condensation product ratio. The methylglyoxal-containing films also present a new peak at $1762 \mathrm{~cm}^{-1}$ that can be assigned to a $\mathrm{C}=\mathrm{O}$ ketone group arising from reaction products of methylglyoxal. Since glyoxal and methylglyoxal do not form films in the absence of propanal, the new ketone peak and the relative increase of the acetal/aldol condensation product ratio must be due to cross-reactions between methylglyoxal and propanal. Another interesting behavior in films that contain methylglyoxal is that the spectra of PM and PGM are very similar, differing only in the intensities of their peaks. This suggests that the presence of methylglyoxal in the organic solutions could inhibit the ability of glyoxal to participate in the formation of films. Such inhibition is also consistent with the observation in Section 3.1 that solutions containing methylglyoxal often formed films more slowly than solutions without it.

${ }^{1} \mathrm{H}$ NMR spectra of the surface films from different organic mixtures are presented in Figures $11(\mathrm{PG}), 12(\mathrm{PM})$, and $13(\mathrm{PGM})$ and compared to the NMR spectrum of the propanal film in Figure 5. All films are 39 days old except for PGM which is 6 days old. 
The ${ }^{1}$ HNMR spectrum of propanal/glyoxal in Figure 11 (39 days old) is similar to the NMR spectrum of propanal in Figure 5 (39 days old) showing peaks corresponding to 2-methyl-2-pentenal (2M2P) and 1,3,5,trimethylbenzene (TMB). Long-chain linear polyacetals (Po) are also possibly present since there are peak groups similar to those previously assigned to Po at 0.94 and 1.0-1.7 ppm. However, when compared with the NMR spectrum of the film formed by propanal (Figure 5), the ${ }^{1} \mathrm{H}$ NMR spectrum of the film formed by propanal/glyoxal (Figure 11) shows weaker peak intensities for 2M2P and TMB relative to the strong peak at $0.94 \mathrm{ppm}$ assigned to the long-chain polymer.

Additionally, new peaks are present at 5.28, 5.80 and $5.88 \mathrm{ppm}$ in the region corresponding to vinylic and conjugated vinylic groups. These new compounds appear to confirm the presence of glyoxal reaction products in the film which are likely due to aldol condensation cross-reaction(s) with propanal since glyoxal does not form films alone. An additional peak at $7.78 \mathrm{ppm}$ in the aromatic region, also not observed previously, further demonstrates the complex chemical composition of this mixed organic film due to crossreactions.

When methylglyoxal is present, the ${ }^{1} \mathrm{HNMR}$ spectra (Figures 12 and 13 for PM and PGM, respectively) display relatively weaker peaks (compared to P and PG) for 2methyl-2-pentenal (2M2P) and 1,3,5-trimethylbenzene (TMB), both products of aldol condensation. Conversely, the intensity of the peaks near $0.9 \mathrm{ppm}$ and $1.6 \mathrm{ppm}$, which were assigned to polyacetals in the propanal-only spectrum, increase in intensity, potentially indicating that relatively greater quantities of polyacetals are formed due to cross-reactions. Although many other compounds could also produce peaks in these 
regions, this interpretation is consistent with the FTIR results. The complexity of the chemical composition of the mixed films is demonstrated by an increasing number of peaks in the region from 5 to $6 \mathrm{ppm}$ going from the propanal/methylglyoxal to the propanal/glyoxal/methylglyoxal film. This region belongs to vinyl and conjugated vinylic groups which shows that aldol condensation cross-reactions also occur. Finally, the ${ }^{1} \mathrm{H}$ NMR spectrum of the PGM film was more similar to PM than to PG, which was also observed in the ATR-FTIR spectra, again indicating that methylglyoxal may suppress film-forming reactions between glyoxal and propanal. In summary, both FTIR and ${ }^{1} \mathrm{H}$ NMR results show strong evidence for products of cross-reactions from acetal formation and aldol condensation reactions and both techniques also suggest that methylglyoxal may inhibit the participation of glyoxal in film-forming reactions.

Propanal/Glyoxal, 48 wt. \% $\mathrm{H}_{2} \mathrm{SO}_{4}$, Day

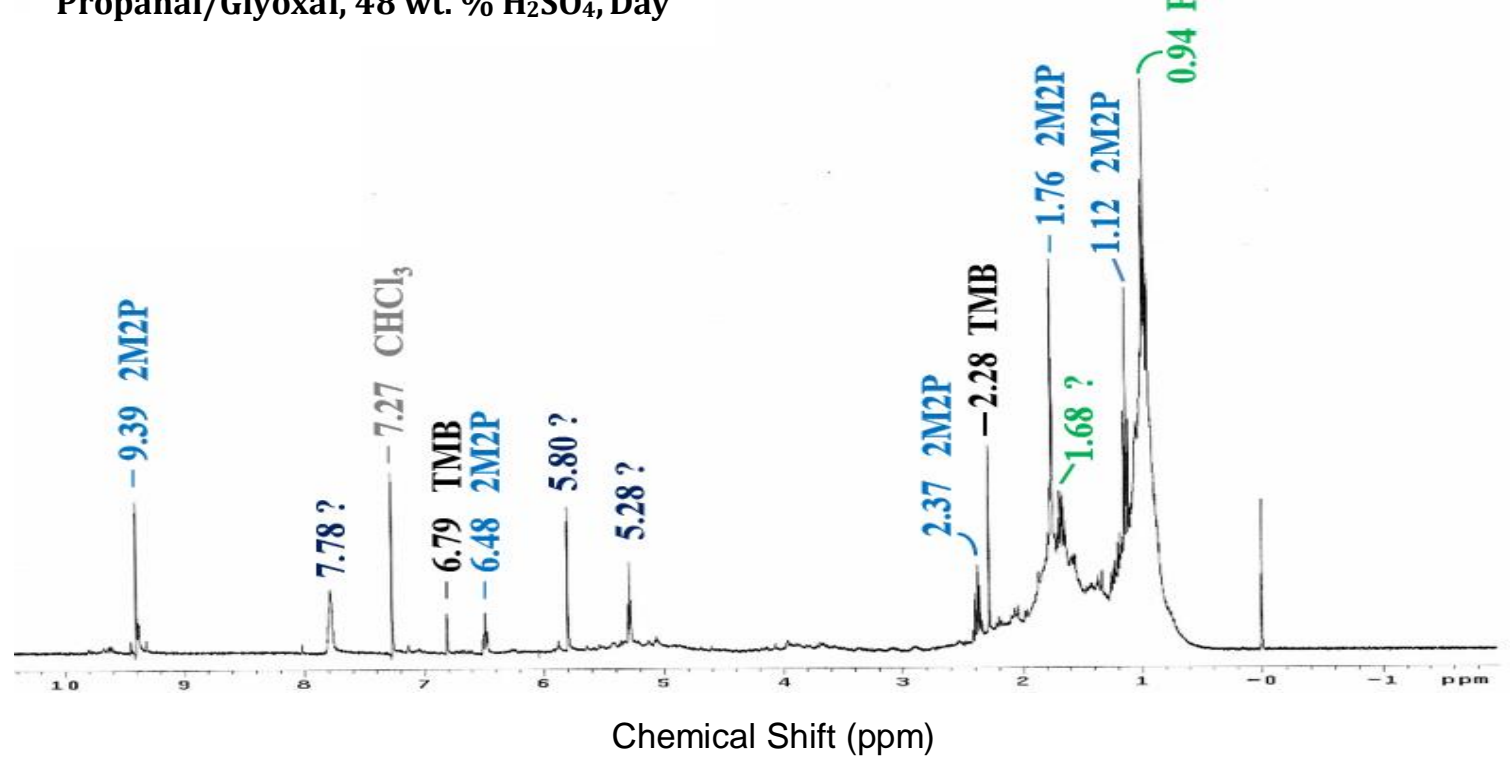

Figure 11: ${ }^{1} \mathrm{H}$ NMR spectrum of a surface film (39 days old) formed on a mixture of propanal and glyoxal in 48 wt. \% $\mathrm{H}_{2} \mathrm{SO}_{4}$ and subsequently dissolved in $\mathrm{CDCl}_{3}$. The solution is $0.30 \mathrm{M}$ in each organic. Assignments to the following species are indicated: 2methyl-2-pentenal (2M2P), 1,3,5-trimethylbenzene (TMB), and "polymer" (Po). 


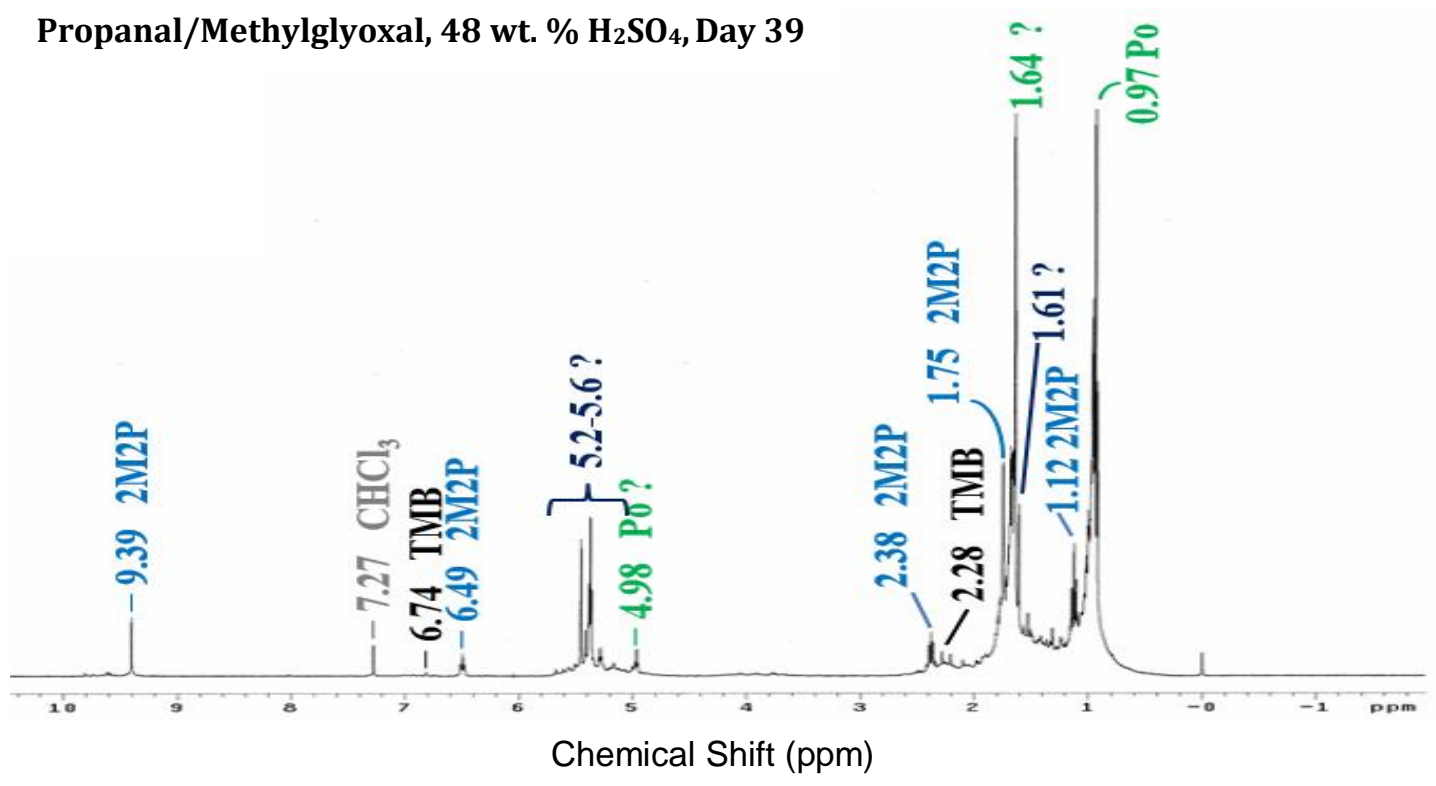

Figure 12: ${ }^{1} \mathrm{H}$ NMR spectrum of a surface film (39 days old) formed on a mixture of propanal and methylglyoxal in $48 \mathrm{wt}$. \% $\mathrm{H}_{2} \mathrm{SO}_{4}$ and subsequently dissolved in $\mathrm{CDCl}_{3}$. The solution is $0.30 \mathrm{M}$ in each organic. Assignments to the following species are indicated: 2-methyl-2-pentenal (2M2P), 1,3,5-trimethylbenzene (TMB), and "polymer" (Po).

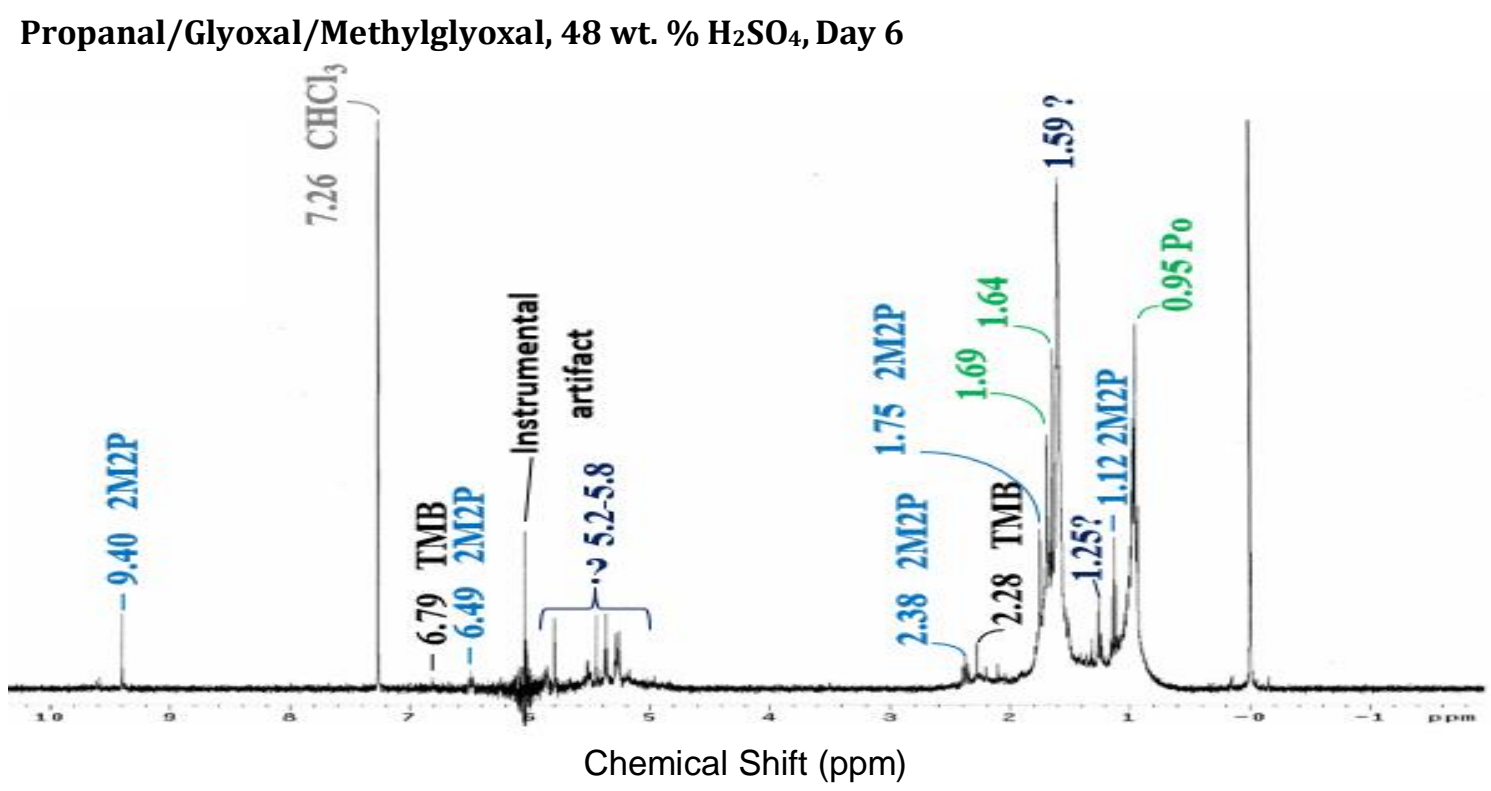

Figure 13: ${ }^{1} \mathrm{H}$ NMR spectrum of a surface film ( 6 days old) formed on a mixture of propanal, glyoxal and methylglyoxal in 48 wt. $\% \mathrm{H}_{2} \mathrm{SO}_{4}$ and subsequently dissolved in $\mathrm{CDCl}_{3}$. The solution is $0.30 \mathrm{M}$ in each organic. Assignments to the following species are indicated: 2-methyl-2-pentenal (2M2P), and 1,3,5-trimethylbenzene (TMB), and "polymer" (Po). 


\subsection{Effect of Aging.}

The change in the chemical composition of films with time is evaluated in this section. The following organic compounds, alone or combined, were used: propanal, propanal/glyoxal, and propanal/methylglyoxal. Films were made from propanal in both 37 and 48 wt. $\% \mathrm{H}_{2} \mathrm{SO}_{4}$ solutions while the remaining organic mixtures were prepared in 48 wt. $\% \mathrm{H}_{2} \mathrm{SO}_{4}$ only. For each of these four mixtures, film samples were collected and analyzed from the same flask at different times after mixing of the solutions.

The effect of aging is evaluated by comparing the ATR-FTIR spectra of films at various ages from 2 to 37 days old presented for each mixture in Figures 14-17. Overall, the FTIR spectra for the films do not show any clear evidence for the formation of any new chemical species as the films age, but the relative peak ratios do change with time. The main changes with age observed in the spectra are changes in the intensities of the peaks in the acetal region $\left(1200-900 \mathrm{~cm}^{-1}\right)$ compared to the $\mathrm{C}=\mathrm{O}$ peak $\left(1690 \mathrm{~cm}^{-1}\right)$ assigned to aldol condensation products.

Beginning with the propanal/48 wt. $\% \mathrm{H}_{2} \mathrm{SO}_{4}$ sample, the ATR-FTIR spectra in Figure 14 show a notable difference between the 2-day-old spectrum (green line) and the older spectra (7, 33 and 37 days old). Specifically, the 2-day-old sample clearly shows the presence of 2,4,6-triethyl-1,3,5-trioxane (T) since its characteristic pattern of bands (Figure 4, orange line) dominate the $1200-900 \mathrm{~cm}^{-1}$ region, while the $\mathrm{T}$ signature is much weaker at 7 days old and finally is nearly obscured by 33 days old. A possible explanation is the formation of longer-chain linear polyacetals that continue to slowly increase in concentration with time while trioxane remains constant (or decreases). 
Alternatively, the evaporation of $\mathrm{T}$ when the flasks are unsealed during sampling could also explain its decreased contribution in older samples. However, the former explanation is favored since it is also consistent with the continued growth of the films with time.

Interestingly, as noted in Section 3.3, when the acidity is decreased from 48 to $37 \mathrm{wt}$. $\% \mathrm{H}_{2} \mathrm{SO}_{4}, 2,4,6$-triethyl-1,3,5-trioxane is very weak or is not present in the FTIR spectrum for the film even in the youngest sample (Figure 15). Also, while the spectrum of the 48 wt. $\% \mathrm{H}_{2} \mathrm{SO}_{4}$ film does not change much after 7 days, the spectrum of the $37 \mathrm{wt}$. $\% \mathrm{H}_{2} \mathrm{SO}_{4}$ film shows a consistent trend with age. Specifically, the peaks in the region assigned to longer-chain linear polyacetals $\left(1200-900 \mathrm{~cm}^{-1}\right)$ increased relative to the $1690 \mathrm{~cm}^{-1}$ peak assigned to aldol condensation products. This may indicate that polyacetal formation reactions go slower relative to aldol condensation reactions in less acidic solutions. The same explanation can be extended to the increasing intensity with age noticed for methyl and methylene peaks $\left(2980-2800 \mathrm{~cm}^{-1}\right)$. Another difference is the small, broad band that increased with time in the $3500-3300 \mathrm{~cm}^{-1}$ alcohol region which likely arises from the alcohol groups on the ends of the polyacetals (Figure 2d), but could also be due to an increase in hemiacetals or single acetals which also contain alcohol groups (Figure 2c). 


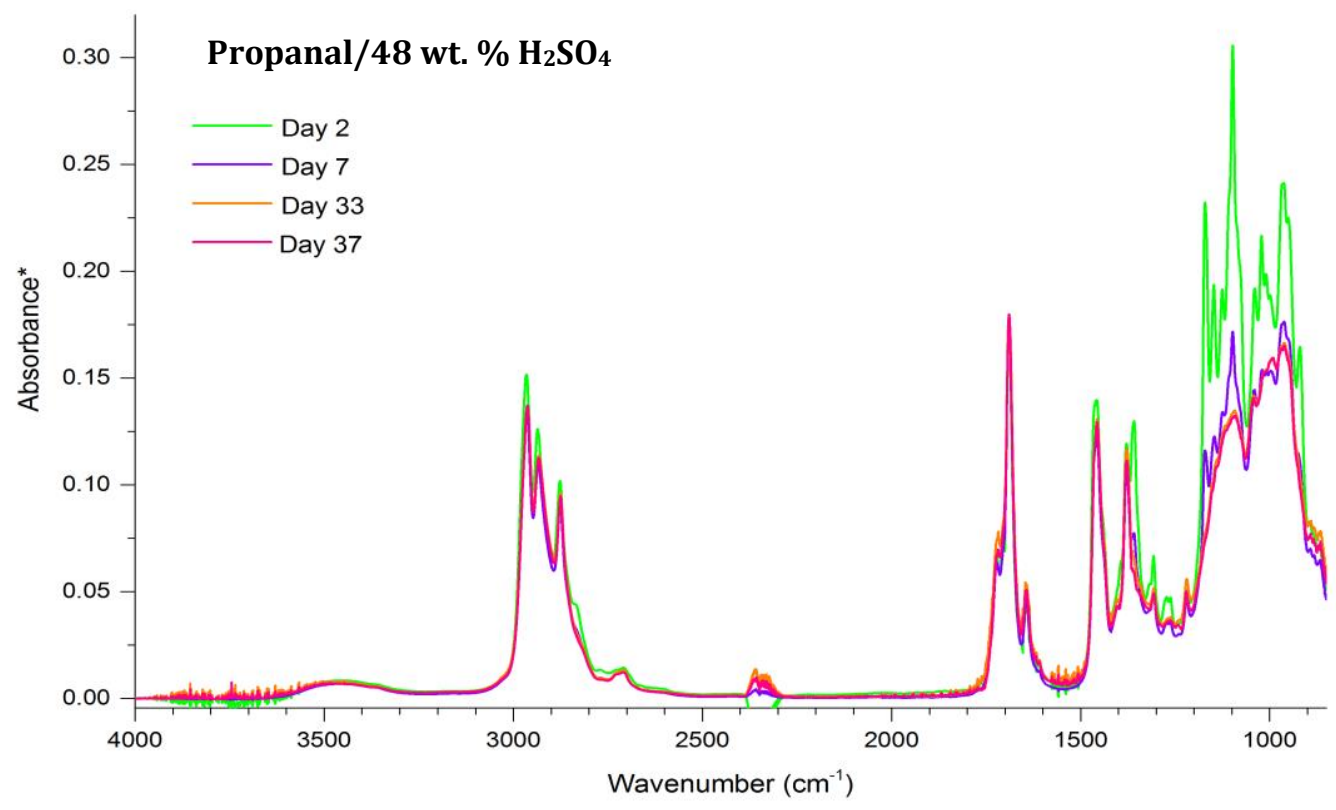

Figure 14: Effect of aging on the ATR-FTIR spectra of a surface film $(2,7,33$, and 37 days old) formed on a mixture of $0.30 \mathrm{M}$ propanal (P) in 48 wt. $\% \mathrm{H}_{2} \mathrm{SO}_{4}$. All spectra were scaled to the carbonyl peak at $1690 \mathrm{~cm}^{-1}$.

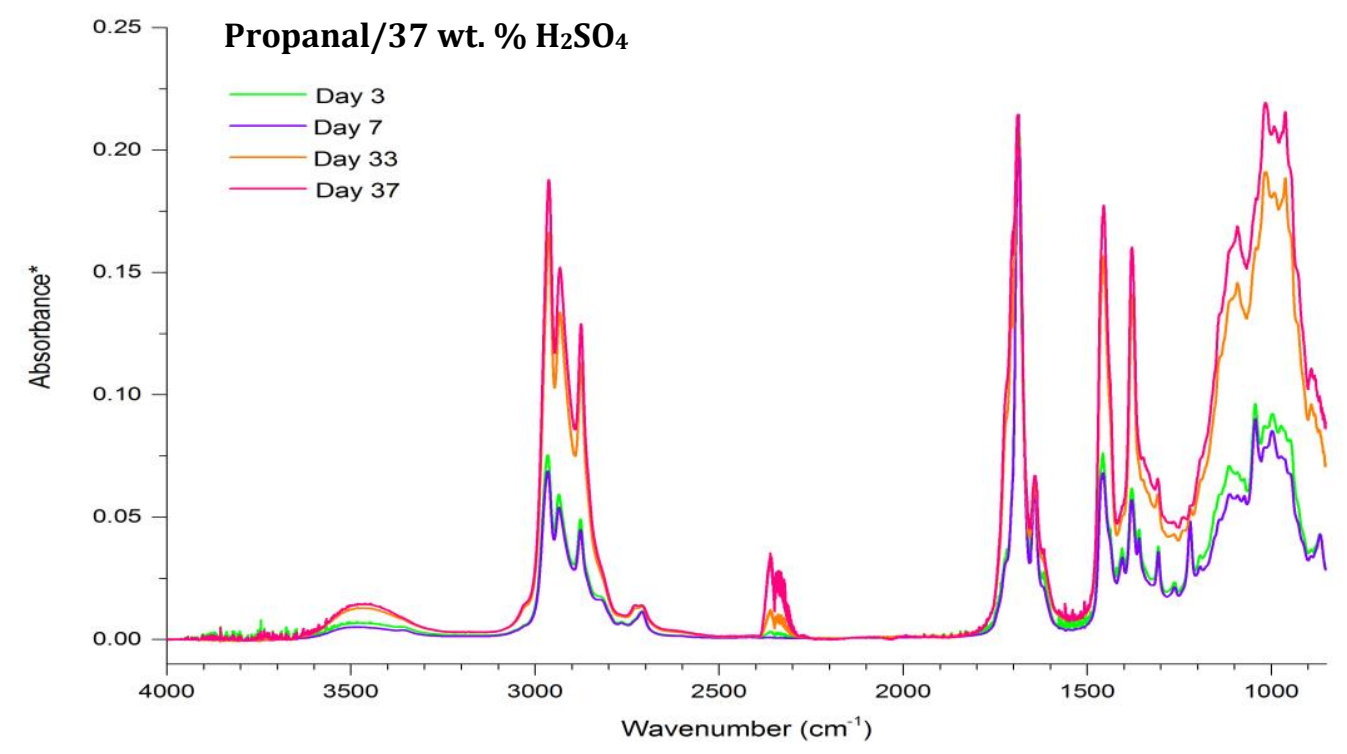

Figure 15: Effect of aging on the ATR-FTIR spectra of a surface film $(3,7,33$, and 37 days old) formed on a mixture of $0.30 \mathrm{M}$ propanal (P) in $37 \mathrm{wt} \% \mathrm{H}_{2} \mathrm{SO}_{4}$. All spectra were scaled to the carbonyl peak at $1690 \mathrm{~cm}^{-1}$.

The effect of aging on the ATR-FTIR spectra of films formed from multiple organics are shown for propanal/glyoxal and propanal/methylglyoxal in Figures 16 and 
17, respectively, both in 48 wt. $\% \mathrm{H}_{2} \mathrm{SO}_{4}$ solution. The aging trend observed for the propanal/glyoxal film (Figure 16) is similar to the trend for the propanal film at $37 \mathrm{wt}$ \% $\mathrm{H}_{2} \mathrm{SO}_{4}$ (Figure 15). Specifically, the spectra show a trend of increasing absorption with time in the $3600-3200 \mathrm{~cm}^{-1}, 3000-2800 \mathrm{~cm}^{-1}$ and $1500-900 \mathrm{~cm}^{-1}$ regions relative to the $1690 \mathrm{~cm}^{-1}$ carbonyl peak, indicating an increase of the polyacetal to aldol condensation product ratio with time. In contrast, the ATR-FTIR spectra of the propanal/methylglyoxal films (Figure 17) show no significant trend with time.

Overall, the trends in chemical composition with age depend on the acidity and the organic mixture and can result in increasing, decreasing or constant ratios between polyacetal and aldol condensation products.

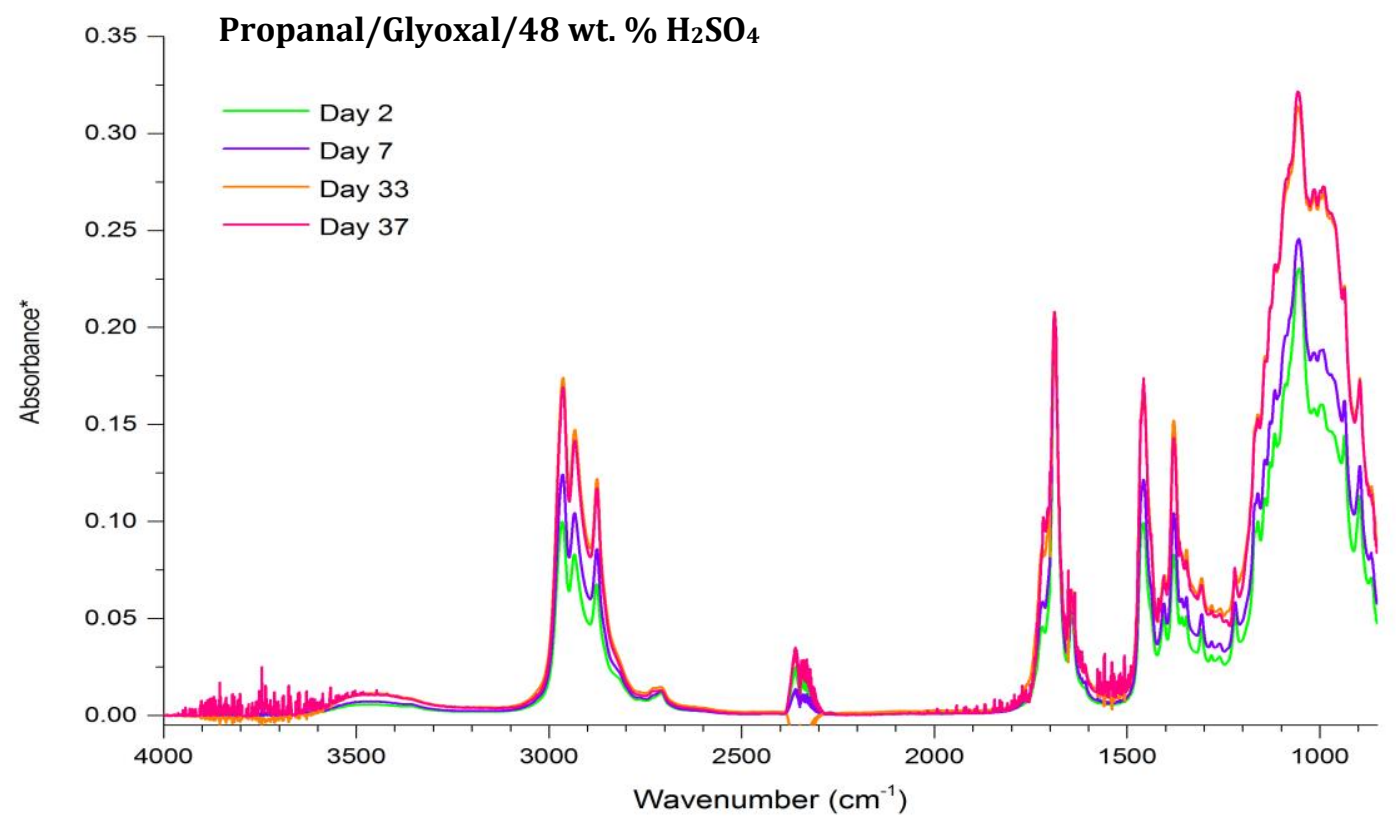

Figure 16: Effect of aging on the ATR-FTIR spectra of a surface film $(2,7,33$, and 37 days old) formed on a mixture of propanal and glyoxal (PG) in 48 wt. \% $\mathrm{H}_{2} \mathrm{SO}_{4}$. The solution is $0.30 \mathrm{M}$ in each organic. All spectra were scaled to the carbonyl peak at 1690 $\mathrm{cm}^{-1}$. 


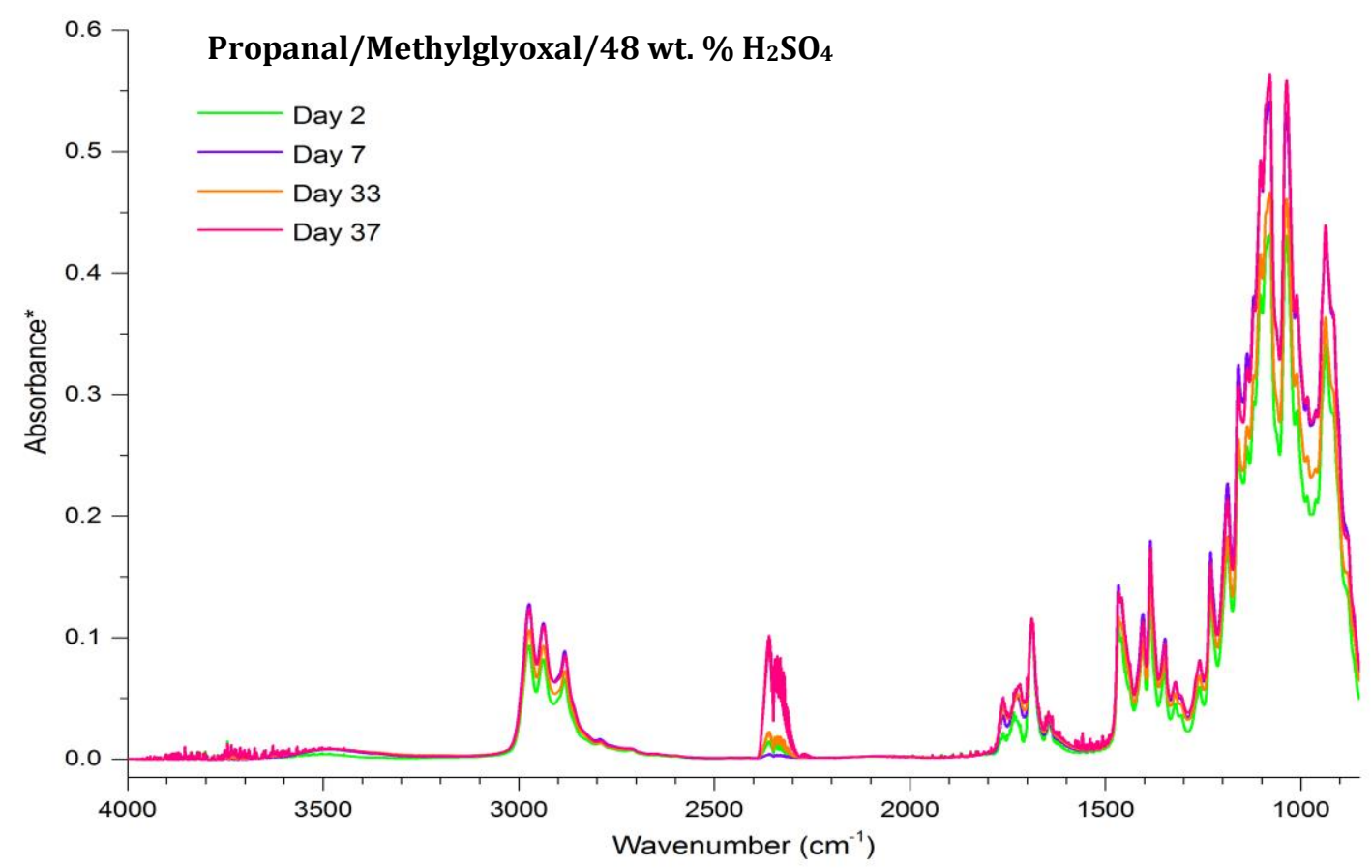

Figure 17: Effect of aging on the ATR-FTIR spectra of a surface film (2, 7, 33, and 37 days old) formed on a mixture of propanal and methylglyoxal (PM) in 48 wt. \% $\mathrm{H}_{2} \mathrm{SO}_{4}$. The solution is $0.30 \mathrm{M}$ in each organic. All spectra were scaled to the carbonyl peak at $1690 \mathrm{~cm}^{-1}$.

\subsection{UV- visible Spectrometric Analysis of Solutions.}

The previous sections presented chemical analysis of colored organic surface films that were formed on acidic solutions. It is also important to evaluate the role and behavior of the organic molecules in the acidic solutions themselves which are also highly colored and could, therefore, impact the radiative properties of UT/LS aerosols.

In order to examine which absorbing species (chromophores) are present and to determine the magnitude of the absorbance that would affect the radiative (absorbing) properties of sulfuric acid aerosols, a series of UV-vis spectra were taken of solutions of propanal, propanal/glyoxal, propanal/methylglyoxal, and propanal/glyoxal/methylglyoxal in 48 and 76 wt.\% $\mathrm{H}_{2} \mathrm{SO}_{4}$ after removal of the solid film fragments by filtering (Figure 
18). These experiments were performed with 274-day-old solutions since lower stratosphere aerosols have long residence times ( 2 years). The solutions were prepared at concentrations of $0.030 \mathrm{M}$ propanal which is ten times more dilute than the surface films work, but more realistic for atmospheric aerosol concentrations.
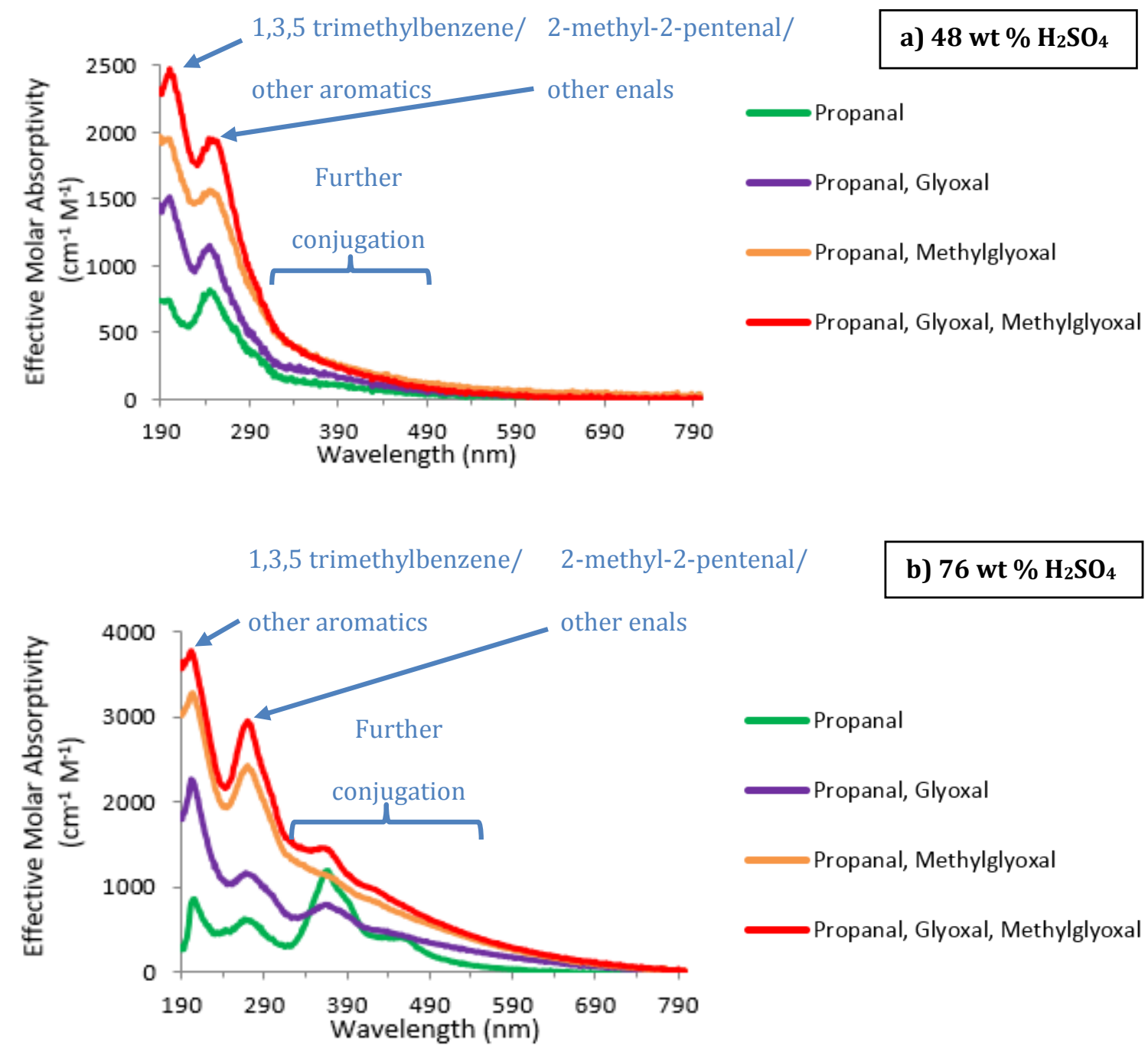

Figure 18: UV-Vis absorption of 274-day-old organic solutions in a) 48 wt. $\% \mathrm{H}_{2} \mathrm{SO}_{4}$ and b) 76 wt. $\% \mathrm{H}_{2} \mathrm{SO}_{4}$, after removal of film/precipitate. Solutions are $0.030 \mathrm{M}$ in each organic. "Effective" molar absorptivity is calculated based only on the concentration of the propanal reactant $(0.030 \mathrm{M})$ so that any changes in absorbance (compared to the propanal-only spectrum) must be due to the presence of the additional organic species. (Adapted with permission from Van Wyngarden et al., Atmos. Chem. Phys., 2015.67) 
Figure 18a shows the UV-vis spectra of propanal and mixtures of propanal with glyoxal and/or methylglyoxal in 48 wt. $\% \mathrm{H}_{2} \mathrm{SO}_{4}$ taken 274 days after mixing. In green, the spectrum of the propanal/sulfuric acid solution shows two peaks at 200 and $245 \mathrm{~nm}$ that most likely correspond to two species also observed in the films, 1,3,5trimethylbenzene and 2-methyl-2-pentenal, which absorb in water at 200 and $234 \mathrm{~nm}$, respectively. The UV-vis spectrum also shows some absorption in the visible region although no distinct peaks were observed. This absorbance probably corresponds to continued aldol condensation of 2-methyl-2-pentenal with propanal and/or itself forming multiple oligomers of various chain lengths which would produce multiple overlapping peaks extending into the visible region. Further evidence for long-chain aldol condensation products is observed in the spectrum of the propanal solution at the higher acidity of 76 wt. $\% \mathrm{H}_{2} \mathrm{SO}_{4}$ (Figure $18 \mathrm{~b}$, green). The most important result is the presence of additional peaks at 365, 388 (shoulder) and $458 \mathrm{~nm}$ extending into the visible region. Their presence can be explained by further acid-catalyzed aldol condensation reactions of 2-methyl-2-pentenal to form oligomers for which each aldol condensation step produces a longer backbone and increasing conjugation.

The UV-vis spectra of mixtures of propanal/glyoxal, propanal/methylglyoxal and propanal/glyoxal/methylglyoxal are also displayed in Figure 18. The "effective" molar absorptivity of the organic mixtures was calculated based only on the concentration of the propanal reactant, so that any changes in absorbance compared to the propanal-only spectrum must be due to the presence of additional organic species. A comparison of the propanal-only spectrum with the spectra of the organic mixtures in 48 wt. $\% \mathrm{H}_{2} \mathrm{SO}_{4}$, 
shows the presence of larger peaks in the mixtures at wavelengths corresponding to $1,3,5$ trimethylbenzene and 2-methyl-2-pentenal which indicates the presence of additional aldol condensation products in the organic mixtures. When the same comparison (propanal-only spectrum against organic mixture spectra) is made at the highest acidity (76 wt. $\% \mathrm{H}_{2} \mathrm{SO}_{4}$ ), the results show a more dramatic increase in the absorbance from aldol condensation products in the mixtures when compared to the propanal-only spectrum. The only exception is that the peak at $365 \mathrm{~nm}$ is less predominant (relative to the rest of the spectrum) in all the organic mixtures than in the propanal-only solution and is actually lower in the propanal/glyoxal mixture than in the propanal-only solution despite the increased organic content. It is possible that non-aldol condensation cross-reactions could be occurring, competing with and decreasing aldol condensation products from propanal. Additionally, cross- or self- aldol condensation reactions of glyoxal and/or methylglyoxal likely result in a mixture of absorbing species that alter the UV-vis spectrum. These results are consistent with evidence for cross-reactions in mixed-organic solutions that was also observed in FTIR and ${ }^{1} \mathrm{H}$ NMR spectra of the surface films. 


\section{CONCLUSIONS AND FURTHER STUDIES}

Organic compounds (propanal, glyoxal and/or methylglyoxal) dissolved in highly concentrated sulfuric acid simulating upper troposphere/lower stratosphere (UT/LS) aerosol conditions, produced colored solutions, precipitates, and surface films. These organic species may influence climate properties of aerosols in the UT/LS if present in sufficient quantities.

This work is focused on the surface films since films on atmospheric aerosols would be expected to control their optical, chemical and cloud forming properties. Therefore, understanding the chemistry involved in the formation of surface films in laboratory experiments would help to understand the role of organic films on acidic UT/LS aerosols and their potential effects on climate. The results presented are based primarily on the surface film made from propanal in 48 wt. \% sulfuric acid. FTIR and ${ }^{1} \mathrm{H}$ NMR spectra of the films formed by propanal in sulfuric acid show spectral features of aldol-condensation products (2-methyl-2-pentenal and 1,3,5-trimethylbenzene), acetals, (long-chain linear polyacetals and 2,4,6-triethyl-1,3,5-trioxane) and propanal itself. The polyacetals are most likely to be responsible for the phase separation. Evidence for crossreactions was present in the FTIR and ${ }^{1} \mathrm{H}$ NMR spectra of films formed on solutions of propanal mixed with glyoxal and/or methylglyoxal, including the observation that increasingly complex film spectra resulted from increasing the number of organic source compounds added to the mixtures. For example, when glyoxal or methylglyoxal was present, the ${ }^{1} \mathrm{H}$ NMR spectra of the films showed additional peaks in the 5.0 to $5.8 \mathrm{ppm}$ region which must be from products of cross-reactions since glyoxal and methylglyoxal 
do not form films without propanal. This result is especially important since crossreactions would be more common than self-reactions in atmospheric aerosols. UV-vis spectra of the solutions also confirm the formation of aldol-condensation products and show evidence of cross-reactions when methylglyoxal and/or glyoxal are present, consistent with the FTIR and ${ }^{1} \mathrm{H}$ NMR results from the surface films.

The formation of films using hydrochloric acid indicates that organosulfates are not necessary for the formation of surface films. Furthermore, the FTIR and ${ }^{1} \mathrm{H}$ NMR spectra of films formed on hydrochloric and sulfuric acid are highly similar, indicating that organosulfates are not major species. However, their presence as minor species cannot be excluded due to the overlapping of peaks from the functional groups of other film species in the FTIR and ${ }^{1} \mathrm{H}$ NMR spectral regions corresponding to sulfates.

Kinetics experiments determined specifically how film formation rates depended on acidity and organic mixture and were consistent with the results from chemical analysis as follows. First, the overall tendency was for films to form faster at high acidity, consistent with acid-catalyzed aldol condensation and acetal formation reactions. Second, the presence of glyoxal in mixtures with propanal seemed to accelerate the formation of films, and cross-reaction with propanal is the likely mechanism since glyoxal alone does not form films.

In conclusion, identification of the major film components as aldol condensation products and acetals indicates that film formation may be important in UT/LS aerosols since many organic species can undergo aldol condensation and acetal formation reactions. Furthermore, the observation of cross-reaction products from both processes 
suggests that reactions of carbonyl species could significantly contribute to aerosol mass and, therefore, affect climate properties since there are many atmospheric organics that may undergo these reactions.

Much work is required to assess the likelihood of the existence of significant films on UT/LS aerosols and to determine the magnitude of climate impacts. To understand the complex chemical composition of the mixed organic films, further studies are needed. An extended new approach could utilize high-performance liquid chromatography (HPLC) with tandem mass spectrometry (MS/MS) to identify the products and to clarify which reactions are the most important for the formation of films from mixtures of multiple organic species. Experiments examining the role of potential gas phase reactants will also be necessary to simulate heterogeneous interactions in the atmosphere. The optical properties of the organic compounds may importantly influence the radiation balance and the climate impact of aerosols under different conditions, so the optical properties of aerosols containing these compounds and films should also be measured. Results from the experiments reported in the present work provide a foundation for these further studies and, ultimately, for understanding of the role of organic reactions in the highlyconcentrated sulfuric acid aerosols in the UT/LS and their impacts on climate. 


\section{REFERENCES}

(1) Jacobson, M.; Hansson, H. C.; Noone, K.; Charlson, R.: Organic atmospheric aerosols: Review and state of the science. Reviews of Geophysics 2000, 38, 267-294.

(2) Kanakidou, M.; Seinfeld, J.; Pandis, S.; Barnes, I.; Dentener, F.; Facchini, M.; Dingenen, R. V.; Ervens, B.; Nenes, A.; Nielsen, C.: Organic aerosol and global climate modelling: a review. Atmospheric Chemistry and Physics 2005, 5, 1053-1123.

(3) Poschl, U.: Atmospheric aerosols: composition, transformation, climate and health effects. Angewandte Chemie International Edition English 2005, 44, 7520-40.

(4) Nienow, A. M.; Roberts, J. T.: Heterogeneous chemistry of carbon aerosols. Annu Rev Phys Chem 2006, 57, 105-28.

(5) Molina, M. J.; Molina, L. T.; Kolb, C. E.: Gas-phase and heterogeneous chemical kinetics of the troposphere and stratosphere. Annu Rev Phys Chem 1996, 47, 327-367.

(6) V. Faye McNeill, N. S., and Allison N. Schwier: Surface-active organics in atmospheric aerosols. Atmospheric and Aerosol Chemistry 2013, 339, 201259.

(7) Murphy, D.; Froyd, K.; Schwarz, J.; Wilson, J.: Observations of the chemical composition of stratospheric aerosol particles. Quarterly Journal of the Royal Meteorological Society 2013.

(8) Stocker, T. F.; Qin, D.; Plattner, G.-K.; Tignor, M.; Allen, S. K.; Boschung, J.; Nauels, A.; Xia, Y.; Bex, V.; Midgley, P. M.: IPCC, 2013. Climate Change 2013: The Physical Science Basis. Contribution of Working Group I to the Fifth Assessment Report of the Intergovernmental Panel on Climate Change Cambridge University Press: Cambridge, United Kingdom and New York, NY, USA, 2013.

(9) Murphy, D.; Thomson, D.; Mahoney, M.: In situ measurements of organics, meteoritic material, mercury, and other elements in aerosols at 5 to 19 kilometers. Science 1998, 282, 1664-1669.

(10) Gettelman, A.; Hoor, P.; Pan, L. L.; Randel, W. J.; Hegglin, M. I.; Birner, T.: The extratropical upper troposphere and lower strathospere. Reviews of Geophysics 2011, 49, RG3003. 
(11) Jacobson, M. Z.: Fundamentals of atmospheric modeling; Cambridge University Press: New York, 2005.

(12) Fueglistaler, S.; Dessler, A. E.; Dunkerton, T. J.; Folkins, I.; Fu, Q.; Mote, P. W.: Tropical tropopause layer. Reviews of Geophysics 2009, 47, RG1004.

(13) Highwood, E. J.; Hoskins, B. J.: The tropical tropopause. Quarterly Journal of the Royal Meteorological Society 1998, 124, 1579-1604.

(14) Atticks, M. G.; Robinson, G. D.: Some features of the structure of the tropical tropopause. Quarterly Journal of the Royal Meteorological Society 1983, 109, 295-308.

(15) Junge, C. E.; Chagnon, C. W.; Manson, J. E.: Stratospheric aerosols. Journal of Meteorology 1961, 18, 81-108.

(16) Rosen, J. M.: The boiling point of stratospheric aerosols. Journal of Applied Meteorology 1971, 10, 1044-1046.

(17) Rosen, J. M.; Hofmann, D. J.; Laby, J.: Stratospheric aerosol measurements II: The worldwide distribution. Journal of the Atmospheric Sciences 1975, $32,1457-1462$.

(18) Steele, H. M.; Hamill, P.: Effects of temperature and humidity on the growth and optical properties of sulphuric acid - water droplets in the stratosphere. Journal of Aerosol Science 1981, 12, 517-528.

(19) Singh, H.; Chen, Y.; Tabazadeh, A.; Fukui, Y.; Bey, I.; Yantosca, R.; Jacob, D.; Arnold, F.; Wohlfrom, K.; Atlas, E.; Flocke, F.; Blake, D.; Blake, N.; Heikes, B.; Snow, J.; Talbot, R.; Gregory, G.; Sachse, G.; Vay, S.; Kondo, Y.: Distribution and fate of selected oxygenated organic species in the troposphere and lower stratosphere over the Atlantic. Journal of Geophysical Research: Atmospheres 2000, 105, 3795-3805.

(20) Zhao, J.; Smith, J. N.; Eisele, F. L.; Chen, M.; Kuang, C.; McMurry, P. H.: Observation of neutral sulfuric acid-amine containing clusters in laboratory and ambient measurements. Atmospheric Chemistry and Physics 2011, 11, 10823-10836.

(21) Hamill, P.; Turco, R.; Toon, O.: On the growth of nitric and sulfuric acid aerosol particles under stratospheric conditions. Journal of Atmospheric Chemistry 1988, 7, 287-315. 
(22) Deshler, T.: Thirty years of in situ stratospheric aerosol size distribution measurements from Laramie, Wyoming $\left(41^{\circ} \mathrm{N}\right)$, using balloon-borne instruments. Journal of Geophysical Research 2003, 108.

(23) Crutzen, P. J.: Albedo enhancement by stratospheric sulfur injections: A contribution to resolve a policy dilemma? Climatic Change 2006, 77, 211220 .

(24) Khalizov, A. F.; Zhang, R.; Zhang, D.; Xue, H.; Pagels, J.; McMurry, P. H.: Formation of highly hygroscopic soot aerosols upon internal mixing with sulfuric acid vapor. Journal of Geophysical Research: Atmospheres 2009, 114.

(25) Tang, I. N.: Chemical and size effects of hygroscopic aerosols on light scattering coefficients. Journal of Geophysical Research: Atmospheres 1996, 101, 19245-19250.

(26) Murphy, D. M.; Cziczo, D. J.; Hudson, P. K.; Thomson, D. S.: Carbonaceous material in aerosol particles in the lower stratosphere and tropopause region. Journal of Geophysical Research 2007, 112.

(27) Froyd, K.; Murphy, D.; Sanford, T.; Thomson, D.; Wilson, J.; Pfister, L.; Lait, L.: Aerosol composition of the tropical upper troposphere. Atmospheric Chemistry and Physics 2009, 9, 4363-4385.

(28) Murphy, D. M.; Cziczo, D. J.; Froyd, K. D.; Hudson, P. K.; Matthew, B. M.; Middlebrook, A. M.; Peltier, R. E.; Sullivan, A.; Thomson, D. S.; Weber, R. J.: Single-particle mass spectrometry of tropospheric aerosol particles. Journal of Geophysical Research 2006, 111.

(29) Nozière, B.; Dziedzic, P.; Córdova, A.: Formation of secondary lightabsorbing "fulvic-like" oligomers: A common process in aqueous and ionic atmospheric particles? Geophysical Research Letters 2007, 34, L21812.

(30) Noziere, B.; Esteve, W.: Organic reactions increasing the absorption index of atmospheric sulfuric acid aerosols. Geophysical Research Letters 2005, 32.

(31) Barsanti, K. C.; Pankow, J. F.: Thermodynamics of the formation of atmospheric organic particulate matter by accretion reactions-Part 1: aldehydes and ketones. Atmospheric Environment 2004, 38, 4371-4382.

(32) Hegarty, A. F.; Dowling, J. P.; Eustace, S. J.; McGarraghy, M.: Enolization of aldehydes and ketones: Structural effects on concerted acid-base catalysis. Journal of the American Chemical Society 1998, 120, 2290-2296. 
(33) Jang, M.; Czoschke, N. M.; Lee, S.; Kamens, R. M.: Heterogeneous atmospheric aerosol production by acid-catalyzed particle-phase reactions. Science 2002, 298, 814-7.

(34) Casale, M.; Richman, A.; Elrod, M.; Garland, R.; Beaver, M.; Tolbert, M.: Kinetics of acid-catalyzed aldol condensation reactions of aliphatic aldehydes. Atmospheric Environment 2007, 41, 6212-6224.

(35) Carey, F. A.; Sundberg, R. J.: Advanced Organic Chemistry: Part A: Structure and Mechanisms; Springer: New York, 2007.

(36) Nozière, B.; Ekström, S.; Alsberg, T.; Holmström, S.: Radical-initiated formation of organosulfates and surfactants in atmospheric aerosols. Geophysical Research Letters 2010, 37, L05806.

(37) Jang, M.; Kamens, R. M.: Atmospheric secondary aerosol formation by heterogeneous reactions of aldehydes in the presence of a sulfuric acid aerosol catalyst. Environmental Science \& Technology 2001, 35, 4758-4766.

(38) Nozière, B.; Esteve, W.: Light-absorbing aldol condensation products in acidic aerosols: Spectra, kinetics, and contribution to the absorption index. Atmospheric Environment 2007, 41, 1150-1163.

(39) Hoang, T. Q.; Zhu, X.; Sooknoi, T.; Resasco, D. E.; Mallinson, R. G.: A comparison of the reactivities of propanal and propylene on HZSM-5. Journal of Catalysis 2010, 271, 201-208.

(40) Garland, R. M.; Elrod, M. J.; Kincaid, K.; Beaver, M. R.; Jimenez, J. L.; Tolbert, M. A.: Acid-catalyzed reactions of hexanal on sulfuric acid particles: Identification of reaction products. Atmospheric Environment 2006, 40, 6863-6878.

(41) Corrochano, P.; García-Río, L.; Poblete, F. J.; Rodríguez-Dafonte, P.: Spontaneous cyclo-trimerization of propionaldehyde in aqueous solution. Tetrahedron Letters 2010, 51, 1761-1765.

(42) Li, Y. J.; Lee, A. K.; Lau, A. P.; Chan, C. K.: Accretion reactions of octanal catalyzed by sulfuric acid: Product identification, reaction pathways, and atmospheric implications. Environmental Science \& Technology 2008, 42, 7138-7145. 
(43) Surratt, J. D.; Gómez-González, Y.; Chan, A. W. H.; Vermeylen, R.; Shahgholi, M.; Kleindienst, T. E.; Edney, E. O.; Offenberg, J. H.; Lewandowski, M.; Jaoui, M.; Maenhaut, W.; Claeys, M.; Flagan, R. C.; Seinfeld, J. H.: Organosulfate Formation in Biogenic Secondary Organic Aerosol. The Journal of Physical Chemistry A 2008, 112, 8345-8378.

(44) Deno, N. C.; Newman, M. S.: Mechanism of Sulfation of Alcohols1,2. Journal of the American Chemical Society 1950, 72, 3852-3856.

(45) Liggio, J.; Li, S.-M.; McLaren, R.: Heterogeneous reactions of glyoxal on particulate matter: Identification of acetals and sulfate esters. Environmental Science \& Technology 2005, 39, 1532-1541.

(46) Iraci, L. T.; Essin, A. M.; Golden, D. M.: Solubility of methanol in lowtemperature aqueous sulfuric acid and implications for atmospheric particle composition. The Journal of Physical Chemistry A 2002, 106, 4054-4060.

(47) Michelsen, R. R.; Staton, S. J. R.; Iraci, L. T.: Uptake and Dissolution of Gaseous Ethanol in Sulfuric Acid. The Journal of Physical Chemistry A 2006, 110, 6711-6717.

(48) Minerath, E. C.; Casale, M. T.; Elrod, M. J.: Kinetics Feasibility Study of Alcohol Sulfate Esterification Reactions in Tropospheric Aerosols. Environmental Science \& Technology 2008, 42, 4410-4415.

(49) Van Loon, L. L.; Allen, H. C.: Methanol Reaction with Sulfuric Acid: A Vibrational Spectroscopic Study. The Journal of Physical Chemistry B 2004, 108, 17666-17674.

(50) Van Loon, L. L.; Allen, H. C.: Uptake and Surface Reaction of Methanol by Sulfuric Acid Solutions Investigated by Vibrational Sum Frequency Generation and Raman Spectroscopies. The Journal of Physical Chemistry A 2008, 112, 7873-7880.

(51) Vinnik, M.; Kislina, I.; Kitaigorodskii, A.; Nikitaev, A.: Kinetics and mechanism of formation and hydrolysis of acid methyl sulfate in aqueous solutions of sulfuric acid. Bulletin of the Academy of Sciences of the USSR Division of Chemical Science 1986, 35, 2447-2453.

(52) Kawamura, K.; Okuzawa, K.; Aggarwal, S.; Irie, H.; Kanaya, Y.; Wang, Z.: Determination of gaseous and particulate carbonyls (glycolaldehyde, hydroxyacetone, glyoxal, methylglyoxal, nonanal and decanal) in the atmosphere at Mt. Tai. Atmospheric Chemistry and Physics 2013, 13, 53695380. 
(53) Volkamer, R.; San Martini, F.; Molina, L. T.; Salcedo, D.; Jimenez, J. L.; Molina, M. J.: A missing sink for gas-phase glyoxal in Mexico City: Formation of secondary organic aerosol. Geophysical Research Letters 2007, 34.

(54) Fu, T.-M.; Jacob, D. J.; Wittrock, F.; Burrows, J. P.; Vrekoussis, M.; Henze, D. K.: Global budgets of atmospheric glyoxal and methylglyoxal and implications for formation of secondary organic aerosols. Journal of Geophysical Research: Atmospheres 2008, 113, D15303.

(55) Tan, Y.; Perri, M. J.; Seitzinger, S. P.; Turpin, B. J.: Effects of Precursor Concentration and Acidic Sulfate in Aqueous Glyoxal-OH Radical Oxidation and Implications for Secondary Organic Aerosol. Environmental Science \& Technology 2009, 43, 8105-8112.

(56) Kawamura, K.; Yasui, O.: Diurnal changes in the distribution of dicarboxylic acids, ketocarboxylic acids and dicarbonyls in the urban Tokyo atmosphere. Atmospheric Environment 2005, 39, 1945-1960.

(57) Ho, K.; Cao, J.; Lee, S.; Kawamura, K.; Zhang, R.; Chow, J. C.; Watson, J. G.: Dicarboxylic acids, ketocarboxylic acids, and dicarbonyls in the urban atmosphere of China. Journal of Geophysical Research: Atmospheres 2007, 112 .

(58) Sareen, N.; Schwier, A.; Shapiro, E.; Mitroo, D.; McNeill, V.: Secondary organic material formed by methylglyoxal in aqueous aerosol mimics. Atmospheric Chemistry and Physics 2010, 10, 997-1016.

(59) Harrick, N.: Surface chemistry from spectral analysis of totally internally reflected radiation. The Journal of Physical Chemistry 1960, 64, 1110-1114.

(60) Fahrenfort, J.: Attenuated total reflection: A new principle for the production of useful infra-red reflection spectra of organic compounds. Spectrochimica Acta 1961, 17, 698-709.

(61) Skoog, D. A.; Holler, F. J.; Nieman, T. A.: Principles of Instrumental Analysis; Thomson Learning: Philadelphia, 1998.

(62) Hind, A. R.; Bhargava, S. K.; McKinnon, A.: At the solid/liquid interface: FTIR/ATR - the tool of choice. Advances in colloid and interface science 2001, 93, 91-114. 
(63) http://www.piketech.com/ATR-Crystal-Selection.html (accessed Feb 28, 2015)

(64) Ha, K.; Perez Montano, S.; Iraci, L.; Van Wyngarden, A.: UV-visible Absorption Properties of Colored Species Formed by Organics in Sulfuric Acid at Concentrations Typical of Upper Troposphere/Lower Stratosphere Aerosols. In AGU Fall Meeting Abstracts, 2011; Vol. 1; pp 0299.

(65) Berry, J.; Perez-Montano, S.; Feick, N.; Ha, K. T.; Leong, L.; Tell, K. A.; Dwisaksono, R.; Khaled, K. A.; Le, H.; Gross, D. S.: Colored solutes, precipitates and surface films produced by reactions of organics in sulfuric acid solutions at upper troposphere/lower stratosphere aerosol acidities. In Abstracts of Papers of the American Chemical Society, 2012; Vol. 243.

(66) Smith, M. B.: March's advanced organic chemistry: reactions, mechanisms, and structure; John Wiley \& Sons: Hoboken, New Jersey, 2013.

(67) Van Wyngarden, A. L.; Pérez-Montaño, S.; Bui, J. V. H.; Li, E. S. W.; Nelson, T. E.; Ha, K. T.; Leong, L.; Iraci, L. T.: Complex chemical composition of colored surface films formed from reactions of propanal in sulfuric acid at upper troposphere/lower stratosphere aerosol acidities. Atmospheric Chemistry and Physics 2015, 15, 4225-4239.

(68) SDBS: Spectral Database for Organic Compounds. SDBSweb, National Institute of Advanced Industrial Science and Technology: http://sdbs.db.aist.go.jp. (accessed Feb 28, 2015)

(69) Bergmann, E. D.; Pinchas, S.: Reaction products of primary $\beta$-hydroxyamines with carbonyl compounds. Recueil des Travaux Chimiques des PaysBas 1952, 71, 161-167.

(70) Novak, A.; Whalley, E.: Infrared spectra and structure of polyaldehydes III. Polyacetaldehyde and polypropionaldehyde. Canadian Journal of Chemistry 1959, 37, 1710-1717.

(71) Novak, A.; Whalley, E.: Infrared spectra and structure of polyaldehydes Part 1.-Polyformaldehyde. Transactions of the Faraday Society 1959, 55, 14841489 .

(72) Novak, A.; Whalley, E.: Infrared spectra and structure of polyaldehydes. Advances in Molecular Spectroscopy; Proceedings of the IVth International Meeting on Molecular Spectroscopy 1962, 1, 907-912. 
(73) Vogl, O.: Polymerization of higher aldehydes. III. Elastomeric polyacetaldehyde. Journal of Polymer Science Part A: General Papers 1964, 2, 4591-4606.

(74) Vogl, O.: Polymerization of higher aldehydes. V. End-capped crystalline isotactic polyaldehydes: Characterization and properties. Journal of Polymer Science Part A: General Papers 1964, 2, 4621-4631.

(75) Pavia, D.; Lampman, G.; Kriz, G.; Vyvyan, J.: Introduction to spectroscopy; Cengage Learning: Belmont, CA, 2008.

(76) Balci, M.: Basic ${ }^{1} H$-and ${ }^{13}$ C-NMR spectroscopy; Elsevier: Amsterdam, 2005.

(77) Silverstein, R.; Webster, F.: Spectrometric identification of organic compounds; John Wiley \& Sons: New Jersey, 2006. 


\section{APPENDIX}

Table A1: FTIR results from surface films formed on 7-day-old mixtures of propanal $(\mathrm{P})$, glyoxal (G) and/or methylglyoxal (M) in 48 wt. $\% \mathrm{H}_{2} \mathrm{SO}_{4}$, compared with neat standards of 2-methyl-2-pentenal (2M2P), 1,3,5-trimethylbenzene (TMB), and 2,4,6-triethyl-1,3,5trioxane $(\mathrm{T})$

\begin{tabular}{|c|c|c|c|c|c|c|c|}
\hline & $\begin{array}{l}\text { Neat T } \\
\left(\mathrm{cm}^{-1}\right)\end{array}$ & $\begin{array}{l}\text { Neat } \\
\text { TMB } \\
\left(\mathrm{cm}^{-1}\right)\end{array}$ & $\begin{array}{l}\text { Neat } \\
2 \mathrm{M} 2 \mathrm{P} \\
\left(\mathrm{cm}^{-1}\right)\end{array}$ & $\begin{array}{l}\text { P film } \\
\left(\mathrm{cm}^{-1}\right)\end{array}$ & $\begin{array}{l}\text { PG film } \\
\left(\mathrm{cm}^{-1}\right)\end{array}$ & $\begin{array}{l}\text { PM film } \\
\left(\mathrm{cm}^{-1}\right)\end{array}$ & $\begin{array}{l}\text { PGM film } \\
\left(\mathrm{cm}^{-1}\right)\end{array}$ \\
\hline $\begin{array}{l}\text { OH stretch } \\
\text { (center) }\end{array}$ & & & 3349 & 3451 & 3482 & 3485 & 3472 \\
\hline $\begin{array}{l}\text { OH stretch } \\
\text { (range) }\end{array}$ & & & & $3289-3618$ & $3286-3641$ & $\begin{array}{c}3291- \\
3623\end{array}$ & $3167-3624$ \\
\hline $\begin{array}{l}(-\mathrm{CH})=\text { alkenes, } \\
\text { vinyl or aromatic }\end{array}$ & & 3011 & 3053 & & & & \\
\hline $\begin{array}{l}\mathrm{CH} 3 \text {, asymm } \\
\text { stretch }\end{array}$ & 2973 & & 2972 & 2963 & 2964 & 2973 & 2972 \\
\hline $\begin{array}{l}\mathrm{CH} 2, \text { asymm } \\
\text { stretch }\end{array}$ & 2940 & 2944 & 2937 & 2934 & 2933 & 2937 & 2936 \\
\hline $\begin{array}{l}\mathrm{CH} 3, \text { symm } \\
\text { stretch }\end{array}$ & 2884 & 2856 & 2878 & 2875 & 2876 & 2882 & 2882 \\
\hline \multirow[t]{2}{*}{$\mathrm{C}-\mathrm{H}$ aldehyde } & 2863 & & 2818 & & & & \\
\hline & & & & & & 2792 & 2790 \\
\hline $\mathrm{C}-\mathrm{H}$ aldehyde & 2772 & & 2767 & & & & \\
\hline $\begin{array}{l}\text { C-H aldehyde, } \\
\text { unbranched }\end{array}$ & 2756 & & & & & & \\
\hline $\begin{array}{l}\text { C-H aldehyde, } \\
\text { branched }\end{array}$ & 2693 & 2733 & 2710 & 2709 & 2708 & & \\
\hline $\begin{array}{l}\mathrm{C}=\mathrm{O} \text { stretch/ } \\
\text { ketone, esters }\end{array}$ & 1745 & 1761 & & & & 1762 & 1760 \\
\hline \multirow[t]{3}{*}{$\begin{array}{l}\mathrm{C}=\mathrm{O} \text { sat. } \\
\text { aldehyde }\end{array}$} & 1717 & 1717 & & 1721 & 1719 & 1724 & 1722 \\
\hline & & & & 1701 & & & \\
\hline & & & 1687 & 1689 & 1688 & 1688 & 1687 \\
\hline $\begin{array}{l}C=C \\
\alpha, \beta-\text { unsaturated }\end{array}$ & & & 1643 & 1643 & 1643 & 1644 & 1643 \\
\hline $\begin{array}{l}C=C \\
\alpha, \beta \text {-unsaturated }\end{array}$ & & 1611 & & & 1617 & & \\
\hline $\begin{array}{l}\mathrm{CH} 3-\mathrm{CH} 2 \\
\text { scissoring }\end{array}$ & 1468 & 1472 & 1460 & & & 1466 & 1465 \\
\hline \multirow[t]{2}{*}{$\begin{array}{l}\mathrm{CH} 3-\mathrm{CH} 2 \\
\text { deformation }\end{array}$} & 1442 & 1444 & & 1456 & 1458 & 1459 & 1458 \\
\hline & 1395 & & 1405 & 1402 & 1405 & 1404 & 1404 \\
\hline
\end{tabular}




\begin{tabular}{|c|c|c|c|c|c|c|c|}
\hline & $\begin{array}{l}\text { Neat T } \\
\left(\mathrm{cm}^{-1}\right)\end{array}$ & $\begin{array}{l}\text { Neat } \\
\text { TMB } \\
\left(\mathrm{cm}^{-1}\right)\end{array}$ & $\begin{array}{l}\text { Neat } \\
2 \mathrm{M} 2 \mathrm{P} \\
\left(\mathrm{cm}^{-1}\right)\end{array}$ & $\begin{array}{l}\text { P film } \\
\left(\mathrm{cm}^{-1}\right)\end{array}$ & $\begin{array}{l}\text { PG film } \\
\left(\mathrm{cm}^{-1}\right)\end{array}$ & $\begin{array}{l}\text { PM film } \\
\left(\mathrm{cm}^{-1}\right)\end{array}$ & $\begin{array}{l}\text { PGM film } \\
\left(\mathrm{cm}^{-1}\right)\end{array}$ \\
\hline $\begin{array}{l}\mathrm{CH} 3 \text { umbrella } \\
\text { bending mode }\end{array}$ & & 1378 & 1381 & 1378 & 1379 & 1385 & 1384 \\
\hline $\begin{array}{l}\text { OH in plane } \\
\text { bend, } \mathrm{S}=\mathrm{O} \\
\text { asymm stretch }\end{array}$ & 1360 & & 1358 & 1359 & 1358 & & \\
\hline $\begin{array}{l}\mathrm{OH} \text { in plane } \\
\text { bend }\end{array}$ & & & & & 1346 & 1347 & 1347 \\
\hline $\begin{array}{l}\text { OH in plane } \\
\text { bend }\end{array}$ & 1318 & & & & & 1320 & 1322 \\
\hline & 1307 & & 1306 & 1307 & 1306 & 1306 & 1306 \\
\hline & 1274 & & & 1271 & 1281 & & \\
\hline $\begin{array}{l}\text { OH deformation, } \\
\text { sulfate }\end{array}$ & 1263 & & 1263 & 1262 & 1260 & 1259 & 1258 \\
\hline & 1239 & & & & & 1230 & 1230 \\
\hline C-C-C ketone & & & 1220 & 1219 & 1219 & & \\
\hline & & & & & & 1188 & 1188 \\
\hline $\begin{array}{l}\mathrm{S}=\mathrm{O} \text { asymm } \\
\text { stretch }\end{array}$ & 1172 & & & 1171 & & & \\
\hline & & 1167 & & & 1160 & 1160 & 1160 \\
\hline & 1149 & & & & & 1137 & 1138 \\
\hline $\mathrm{C}-\mathrm{O}$ or $\mathrm{C}-\mathrm{C}-\mathrm{C}$ & 1126 & & & 1124 & 1128 & & \\
\hline $\mathrm{C}-\mathrm{O}$ or $\mathrm{C}-\mathrm{C}-\mathrm{C}$ & & & 1112 & & 1116 & 1120 & 1117 \\
\hline & & & & & & 1103 & 1102 \\
\hline & & & & 1107 & & & \\
\hline & 1099 & & & 1098 & & & 1102 \\
\hline & & & & 1086 & 1087 & 1088 & 1088 \\
\hline$=\mathrm{C}-\mathrm{H},=\mathrm{CH} 2$ & & & 1072 & 1075 & 1077 & 1081 & 1077 \\
\hline & & & & & & 1065 & \\
\hline $\begin{array}{l}\text { C-C-O stretch, } \\
\mathrm{OH} \text { asymm } \\
\text { stretch }\end{array}$ & & & & & 1054 & & \\
\hline & 1039 & 1039 & 1043 & 1040 & & 1036 & 1037 \\
\hline & 1023 & & & 1020 & 1016 & & \\
\hline & 1010 & & & 1008 & & 1010 & 1010 \\
\hline$=\mathrm{C}-\mathrm{H},=\mathrm{CH} 2$ & 999 & & 998 & 996 & 991 & & \\
\hline$=\mathrm{C}-\mathrm{H},=\mathrm{CH} 2$ & & & & & & 983 & 981 \\
\hline$=\mathrm{C}-\mathrm{H},=\mathrm{CH} 2$ & 968 & & & 961 & & 961 & \\
\hline & 963 & & & 951 & & & \\
\hline & & & & & 935 & 937 & 935 \\
\hline
\end{tabular}




\begin{tabular}{|c|c|c|c|c|c|c|c|}
\hline & $\begin{array}{l}\text { Neat T } \\
\left(\mathrm{cm}^{-1}\right)\end{array}$ & $\begin{array}{l}\text { Neat } \\
\text { TMB } \\
\left(\mathrm{cm}^{-1}\right)\end{array}$ & $\begin{array}{c}\text { Neat } \\
2 \mathrm{M} 2 \mathrm{P} \\
\left(\mathrm{cm}^{-1}\right)\end{array}$ & $\begin{array}{l}\text { P film } \\
\left(\mathrm{cm}^{-1}\right)\end{array}$ & $\begin{array}{l}\text { PG film } \\
\left(\mathrm{cm}^{-1}\right)\end{array}$ & $\begin{array}{l}\text { PM film } \\
\left(\mathrm{cm}^{-1}\right)\end{array}$ & $\begin{array}{l}\text { PGM film } \\
\left(\mathrm{cm}^{-1}\right)\end{array}$ \\
\hline & & 928 & & 924 & & & 918 \\
\hline$=\mathrm{C}-\mathrm{H},=\mathrm{CH} 2$ & 919 & & 912 & & & 919 & \\
\hline$=\mathrm{C}-\mathrm{H},=\mathrm{CH} 2$ & & & & 892 & 896 & & 901 \\
\hline $\begin{array}{l}\mathrm{C}-\mathrm{C}-\mathrm{O} \text { symm } \\
\text { stretch, =C-H, } \\
=\mathrm{CH} 2\end{array}$ & & 883 & & 883 & & 884 & \\
\hline S-O stretch & & & 865 & 867 & 866 & 864 & \\
\hline & & 833 & & 834 & 832 & & \\
\hline
\end{tabular}


Table A2 : ${ }^{1} \mathrm{H}$ NMR results from surface films formed on mixtures of propanal (P), glyoxal (G) and/or methylglyoxal(M) compared with neat standards of 2-methyl-2pentenal (2M2P), 1,3,5 trimethylbenzene (TMB), 2,4,6-triethyl-1,3,5-trioxane (T) and propanal $(\mathrm{P})$. Chemical shift ranges are shown for different types of protons with specific values for our standards indicated in bold.

\begin{tabular}{|c|c|c|c|c|c|c|c|}
\hline Group & $\begin{array}{c}\text { Chemical } \\
\text { Shift } \\
\text { (ppm) }\end{array}$ & $\begin{array}{c}\mathrm{P} \text { film } \\
37 \mathrm{wt} . \% \\
\mathrm{H}_{2} \mathrm{SO}_{4} \\
36 \text { days old } \\
\text { (ppm) }\end{array}$ & $\begin{array}{c}\mathrm{P} \text { film } \\
48 \mathrm{wt} . \% \\
\mathrm{H}_{2} \mathrm{SO}_{4} \\
39 \text { days old } \\
\text { (ppm) }\end{array}$ & $\begin{array}{c}\text { PG film } \\
48 \text { wt. \% } \\
\mathrm{H}_{2} \mathrm{SO}_{4} \\
39 \text { days old } \\
\text { (ppm) }\end{array}$ & $\begin{array}{l}\text { PGM film } \\
48 \text { wt. \% } \\
\mathrm{H}_{2} \mathrm{SO}_{4} \\
6 \text { days old } \\
\text { (ppm) }\end{array}$ & $\begin{array}{c}\text { PM film } \\
48 \text { wt. \% } \\
\mathrm{H}_{2} \mathrm{SO}_{4} \\
39 \text { days old } \\
\text { (ppm) }\end{array}$ & $\begin{array}{c}\text { P film } \\
\mathrm{HCl} \\
6.77 \mathrm{M} \\
6 \text { days old } \\
\text { (ppm) }\end{array}$ \\
\hline & & & & & 0.92 & & \\
\hline $\mathrm{RCH} 3$ & $0.70-1.30$ & & & & 0.93 & & \\
\hline \multirow[t]{7}{*}{$\mathbf{T}$} & $(0.94)$ & 0.95 & 0.94 & 0.94 & 0.95 & & \\
\hline & & & 0.96 & & & & \\
\hline & & 0.97 & 0.97 & & & 0.97 & 0.96 \\
\hline & & & 0.98 & & & & \\
\hline & & 0.99 & & & & & \\
\hline & & 1.03 & 1.00 & 1.01 & & 1.02 & \\
\hline & & 1.06 & & & & & 1.05 \\
\hline \multirow[t]{3}{*}{$\mathrm{P} / 2 \mathrm{M} 2 \mathrm{P}$} & (1.10/1.11) & 1.12 & 1.11 & 1.12 & 1.12 & 1.12 & 1.12 \\
\hline & & 1.16 & & & & & \\
\hline & & 1.18 & & & & & \\
\hline \multirow[t]{6}{*}{$\mathrm{R} 2 \mathrm{CH} 2$} & $1.20-1.40$ & 1.20 & & & & & \\
\hline & & 1.22 & & & 1.22 & & \\
\hline & & 1.24 & & & 1.25 & & 1.24 \\
\hline & & & & & 1.31 & 1.30 & \\
\hline & & & & 1.32 & & & \\
\hline & & & & 1.36 & & & \\
\hline \multirow[t]{6}{*}{$\mathrm{R} 3 \mathrm{CH}$} & $1.40-1.70$ & & & & & & \\
\hline & & & & & & 1.50 & \\
\hline & & & & & 1.59 & 1.61 & 1.60 \\
\hline & & & 1.62 & & & & \\
\hline & & 1.64 & & & 1.64 & 1.64 & \\
\hline & & & & & & 1.67 & \\
\hline $\mathbf{T}$ & (1.69) & & & 1.68 & 1.69 & & \\
\hline \multirow[t]{3}{*}{$2 \mathrm{M} 2 \mathrm{P}$} & $(1.74)$ & 1.74 & 1.74 & 1.76 & 1.75 & 1.75 & 1.75 \\
\hline & & 1.96 & & & & & \\
\hline & & 1.97 & & & & & \\
\hline
\end{tabular}




\begin{tabular}{|c|c|c|c|c|c|c|c|}
\hline Group & $\begin{array}{c}\text { Chemical } \\
\text { Shift }\end{array}$ & $\begin{array}{c}\text { P film } \\
37 \text { wt. \% } \\
\mathrm{H}_{2} \mathrm{SO}_{4} \\
36 \text { days old }\end{array}$ & $\begin{array}{c}\text { P film } \\
48 \text { wt. \% } \\
\mathrm{H}_{2} \mathrm{SO}_{4} \\
39 \text { days old }\end{array}$ & $\begin{array}{c}\text { PG film } \\
48 \text { wt. \% } \\
\mathrm{H}_{2} \mathrm{SO}_{4} \\
39 \text { days old }\end{array}$ & $\begin{array}{c}\text { PGM film } \\
48 \text { wt. \% } \\
\mathrm{H}_{2} \mathrm{SO}_{4} \\
6 \text { days old }\end{array}$ & $\begin{array}{c}\text { PM film } \\
48 \text { wt. \% } \\
\mathrm{H}_{2} \mathrm{SO}_{4} \\
39 \text { days old }\end{array}$ & $\begin{array}{c}\text { P film } \\
\mathrm{HCl} \\
6.77 \mathrm{M} \\
6 \text { days old } \\
\end{array}$ \\
\hline $\begin{array}{l}\mathrm{HC}-\mathrm{C}=\mathrm{O} \\
\text { Carbonyl }\end{array}$ & $2.00-2.70$ & & & & & & 2.04 \\
\hline \multirow[t]{3}{*}{ Ketone } & $2.10-2.40$ & 2.15 & & & 2.10 & & \\
\hline & & & & & 2.20 & & \\
\hline & & 2.28 & 2.28 & 2.28 & 2.28 & 2.28 & 2.28 \\
\hline TMB & (2.36) & 2.38 & 2.38 & 2.37 & 2.38 & 2.38 & 2.38 \\
\hline $\mathbf{P}$ & (2.47) & & 2.47 & & & & \\
\hline$\left(\mathrm{OSO}_{2} \mathrm{R}\right)$ & $3.00-3.19$ & & & & & & \\
\hline Ethers & $3.00-3.4$ & & & & & & \\
\hline \multirow[t]{9}{*}{ Vinylic } & $4.60-5.90$ & & & & 4.96 & & \\
\hline & & & & & & 4.98 & \\
\hline & & & & & & 5.00 & \\
\hline & & & & & 5.17 & & \\
\hline & & & & & 5.25 & & \\
\hline & & & & 5.28 & 5.28 & 5.29 & \\
\hline & & & & & 5.37 & 5.36 & \\
\hline & & & & & 5.41 & 5.40 & \\
\hline & & & & & 5.45 & 5.45 & \\
\hline \multirow[t]{6}{*}{$\begin{array}{c}\text { Vinylic } \\
\text { Conj. }\end{array}$} & $5.50-7.50$ & & & & 5.51 & 5.50 & \\
\hline & & & & & & 5.56 & \\
\hline & & & & & & 5.60 & \\
\hline & & & & & & 5.65 & \\
\hline & & & & 5.80 & & & \\
\hline & & 5.90 & & 5.88 & & & \\
\hline Aromatic & $6.00-8.50$ & & & & 6.04 & & \\
\hline \multirow[t]{2}{*}{$2 M 2 P$} & $(6.48)$ & 6.48 & 6.48 & 6.48 & 6.49 & 6.49 & 6.48 \\
\hline & & 6.72 & & & & & \\
\hline \multirow[t]{3}{*}{ TMB } & (6.79) & 6.80 & 6.79 & 6.79 & 6.79 & 6.74 & 6.80 \\
\hline & & 7.00 & & & & & \\
\hline & & 7.17 & & 7.15 & & & \\
\hline \multirow[t]{3}{*}{$\mathrm{CDCl} 3$} & 7.24 & 7.28 & 7.27 & 7.27 & 7.26 & 7.27 & 7.27 \\
\hline & & & & 7.78 & & & \\
\hline & & & 8.02 & 8.04 & & & \\
\hline \multirow[t]{3}{*}{ Aldehyde } & 9-10.0 & 9.18 & & & & & \\
\hline & & 9.32 & 9.30 & 9.32 & & & \\
\hline & & & & 9.38 & & & \\
\hline
\end{tabular}




\begin{tabular}{|c|c|c|c|c|c|c|c|}
\hline Group & $\begin{array}{c}\text { Chemical } \\
\text { Shift }\end{array}$ & $\begin{array}{c}\text { P film } \\
37 \text { wt. \% } \\
\mathrm{H}_{2} \mathrm{SO}_{4} \\
36 \text { days old }\end{array}$ & $\begin{array}{c}\text { P film } \\
48 \text { wt. \% } \\
\mathrm{H}_{2} \mathrm{SO}_{4} \\
39 \text { days old }\end{array}$ & $\begin{array}{c}\text { PG film } \\
48 \text { wt. \% } \\
\mathrm{H}_{2} \mathrm{SO}_{4} \\
39 \text { days old } \\
\end{array}$ & $\begin{array}{c}\text { PGM film } \\
48 \text { wt. \% } \\
\mathrm{H}_{2} \mathrm{SO}_{4} \\
6 \text { days old }\end{array}$ & $\begin{array}{c}\text { PM film } \\
48 \text { wt. \% } \\
\mathrm{H}_{2} \mathrm{SO}_{4} \\
39 \text { days old }\end{array}$ & $\begin{array}{c}\text { P film } \\
\mathrm{HCl} \\
6.77 \mathrm{M} \\
6 \text { days old } \\
\end{array}$ \\
\hline \multirow[t]{7}{*}{$2 \mathrm{M} 2 \mathrm{P}$} & (9.39) & 9.39 & 9.39 & 9.39 & 9.40 & 9.39 & 9.40 \\
\hline & & & 9.45 & 9.45 & & & \\
\hline & & & 9.59 & & 9.58 & & \\
\hline & & & 9.62 & 9.60 & 9.62 & 9.60 & \\
\hline & & & 9.65 & & & & \\
\hline & & & 9.75 & & & 9.74 & \\
\hline & & & 9.79 & 9.80 & & 9.80 & \\
\hline
\end{tabular}

